\title{
Nacionalismo y desarrollo: una alternativa para México
}

\author{
Gaspar Núñez Rodríguez \\ y José Antonio Romero Tellaeche
}

\section{Resumen}

Los países más desarrollados han contado, generalmente, con una activa participación del Estado, un Estado impulsor, regulador, proteccionista, entre otros roles, aunque el concepto que más se ha consolidado es, al parecer, el de un Estado desarrollador. Se construye en este trabajo una matriz de contabilidad social de México para diseñar un modelo dinámico-recursivo de equilibrio general aplicado, a fin de analizar y cuantificar el impacto de políticas que serían impulsadas por el Estado; en particular, políticas dirigidas a un incremento del ahorro privado y subsidios al consumo de insumos nacionales. Se encontró que la aplicación de estas sencillas políticas tiene en general un sustancial impacto positivo, de lo que se desprenden importantes elementos de política económica para una estrategia de desarrollo.

\section{Palabras clave}

Nacionalismo, desarrollo económico, desarrollo social, política económica, estrategias del desarrollo, modelos econométricos, contabilidad social, México

\section{Clasificación JEL}

C68, D58, E16, O21, O41, O54

\section{Autores}

Gaspar Núñez Rodríguez es Investigador del Programa de Análisis Económico de México (PRAEM) del Centro de Estudios Económicos (CEE) de El Colegio de México. Correo electrónico: gaspar.nunez@colmex.mx.

José Antonio Romero Tellaeche es Profesor Investigador del Centro de Estudios Económicos (CEE) de El Colegio de México. Correo electrónico: jromero@colmex.mx. 


\section{Introducción}

El estancamiento económico y la creciente desigualdad que ha experimentado México en las últimas tres décadas han llevado a muchos estudiosos a proponer soluciones simplistas para salir del atraso, como lo ilustra la idea que se ha reiterado de forjar un México justo e incluyente (CCO Noticias, 2015). Sin embargo, los países que han logrado un crecimiento acelerado han motivado a su población a trabajar más y hacer mayores sacrificios sobre la base de un fuerte nacionalismo.

Hirschman (1968) identifica el nacionalismo como el elemento "aglutinante" del desarrollo del Asia oriental en el contexto de "desarrollo tardío"; no se trata de un nacionalismo injustificado, sino de uno que surgió como reacción a la guerra y al imperialismo, y que se manifestó de diferentes maneras: con el comunismo en China y la República Popular Democrática de Corea y el Estado desarrollador capitalista en el Japón, la República de Corea y la provincia china de Taiwán. También por esta razón los Estados desarrolladores del Asia oriental tienen más en común con las naciones europeas de desarrollo tardío de finales del siglo XIX, como Alemania, y menos con las sociedades en desarrollo contemporáneas de América Latina y otras regiones (Greenfeld, 2005; Hirschman, 1961 y 1968; Johnson, 1982; Woo-Cumings, 1999).

El nacionalismo contribuye al impulso inicial del desarrollo y, a su vez, el primero se fortalece. Para crecer, México debe recurrir una vez más al nacionalismo y elevar su autoestima; se debe aspirar a ser más que solo "un país más justo e incluyente" (CCO Noticias, 2015). La mayoría de los países que han logrado bienestar económico no se lo han planteado como meta, sino que lo han conseguido como un subproducto del desarrollo. México tiene el tamaño y los elementos para convertirse en una potencia media; con una población de poco más de 100 millones de habitantes, puede aspirar a situarse en un lugar destacado de la comunidad internacional, en el mismo nivel que países como el Japón, la Federación de Rusia o Alemania, todos con población de magnitud similar a la suya. La forma de lograrlo es planificando el desarrollo, como lo han hecho los países exitosos.

Para ello, no es necesario inventar algo nuevo. Basta echar una mirada a países que lograron desarrollarse en unas cuantas décadas: el caso de Alemania en el siglo XIX (Lee, 1991; Pierenkemper y Tilly, 2004), el Japón a principios del siglo XX, la República de Corea y la provincia china de Taiwán en la segunda mitad del siglo XX, China a fines del siglo XX y principios del siglo XXI y, más recientemente, Viet Nam. Al estudiar a estos países, se encuentra que en todos hubo una intención verdadera de desarrollarse y que planificaron su economía para acelerar el desarrollo y alcanzar a los países líderes en unas cuantas décadas. En todos ellos existió un Estado desarrollador, que se define como aquel que busca el desarrollo capitalista acelerado mediante la planificación a través de un plan racional consensuado, que combina la propiedad privada con la dirección del Estado, esto es, un capitalismo administrado (Amsden, 1992; Chibber, 2014; Evans, 1995; Kasahara, 2013; Kim, 1999; Kristof y WuDunn, 2000; Leftwich, 1994 y 1995; Maman y Rosenhek, 2011; Minns, 2001; Nabi y Shivakumar, 2001; Önis, 1991; Polidano, 2001; Thompson, 1996; Vogel, 1991; Wade, 2003; Wan, 2008; Weiss, 2000; Wong, 2004; Woo-Cumings, 1999).

Los componentes estilizados de esta estrategia incluyen: i) un Estado fuerte; ii) un nacionalismo capaz de llevar a la población a sacrificios presentes para lograr el futuro desarrollo del país; iii) una burocracia eficiente y, dentro de ella, un cuerpo de élite con amplios poderes de planificación económica 
y social ${ }^{1}$; iv) políticas de fomento del ahorro (o desincentivos del consumo) ${ }^{2}$; v) exclusión de la inversión extranjera o aceptación de una muy regulada, como en el caso de China (Alfaro y Charlton, 2013; Chang, 2003; Rodríguez y Rodrik, 1999; Paul, 1979); vi) un sistema financiero fuerte, controlado por el Estado, para canalizar el ahorro nacional y los préstamos extranjeros a sectores estratégicos (Skocpol, 2014; Chandrasekhar, 2013; Rashid, 2013; Woo-Cumings, 1999; Johnson, 2015; Zysman, 1983)3; vii) la selección de sectores estratégicos en función de su intensidad de capital, rendimientos crecientes ${ }^{4}$, economías de aprendizaje e innovación, entre otras características ${ }^{5}$, y con alta elasticidad de la demanda respecto del ingreso ${ }^{6}$; viii) sectores claves con protección arancelaria, acceso a divisas y apoyos financieros en función de su desempeño (Aoki, Kim y Okuno-Fujiwara, 2000); ix) política educativa y científica y tecnológica en función de estos objetivos ${ }^{7}$, y x) utilización del tipo de cambio para hacer más competitiva la economía (Rodrik, 2007).

Ninguno de los componentes señalados puede considerarse en forma aislada, sino como parte de la estrategia global; es decir, ninguna política tiene sentido por sí sola, su relevancia estriba en que contribuye a lograr el objetivo último, que es la grandeza de la nación.

Para analizar los efectos potenciales de la posible adopción de un modelo de Estado desarrollador en México, diseñamos un modelo dinámico-recursivo de equilibrio general aplicado (MEGA), que se basa en una matriz de contabilidad social, construida a partir de la "Matriz de Insumo Producto 2012", publicada por el Instituto Nacional de Estadística y Geografía (INEGI, 2016), e información adicional de las cuentas nacionales. Se trata de un modelo que considera consumidores, Gobierno, factores, sectores productivos y sector externo. Los consumidores y el Gobierno consumen bienes nacionales e importados y también ahorran. Las empresas utilizan trabajo, capital y bienes intermedios para producir el bien final. Los bienes intermedios son nacionales e importados. El sistema de precios y el nivel de ingreso motivan las decisiones de todos los agentes económicos. La característica primordial del modelo es la inclusión de rendimientos crecientes, que constituye el núcleo que da sentido a la estrategia del modelo de Estado desarrollador.

1 En el Japón, después de la Segunda Guerra Mundial, se restableció un fuerte vínculo entre el Gobierno y las grandes empresas. La burocracia y el sector privado se combinaron para establecer dicho vínculo y por medio de este, el Gobierno implementó un sistema de dirección administrativa con departamentos gubernamentales claves, como el Ministerio de Comercio Internacional y de Industria (MITI) y el Ministerio de Finanzas (MOF), que participaron activamente protegiendo y subsidiando empresas cuyas actividades se ajustaban a las prioridades nacionales. El equivalente del MITI japonés en la República de Corea fue el Ministerio de Comercio e Industria (MTI), institución que contribuyó a crear e implementar las políticas industriales del Gobierno de ese país (Chen, 1995).

2 Gran parte del éxito de los países asiáticos se atribuye a la tradicional ética confuciana, que exalta cualidades como la diligencia, la frugalidad, la solidaridad familiar, la armonía del grupo y el énfasis en la educación. Para otros países en desarrollo, estas enseñanzas sirven como recordatorio de que, si quieren aplicar las lecciones de las experiencias de Asia oriental, no es suficiente imitar simplemente los aspectos económicos e institucionales de estas, sino que también deben considerar en profundidad los rasgos culturales, que desempeñaron un papel fundamental en el éxito de Asia oriental. Esto no quiere decir que estos países deban adoptar la tradición confuciana. Más bien, hay que tener presentes los efectos benéficos que aportan ciertos rasgos culturales propios de cada país, sacar provecho de ellos y fomentarlos para lograr los propósitos deseados (Liang, 2010). Asimismo, la puesta en marcha de un plan de pensiones en Singapur, basada en contribuciones individuales, fomentó importantes incrementos del ahorro (Monetary Authority of Singapore, 1991).

3 Al respecto, Skocpol (2014) sostiene: "A todos estos tipos de preguntas debe responderse en cualquier estudio de las capacidades del Estado. Los medios del Estado para captar y emplear recursos financieros nos dicen más que cualquier otro factor individual sobre sus capacidades actuales (e inmediatamente posibles) para crear o reforzar sus organizaciones, para contratar personal, para lograr el apoyo político, para subvencionar iniciativas económicas y para financiar programas sociales" (págs. 108 y 109).

4 Véase una excelente presentación sobre la importancia de los rendimientos crecientes en Young (2009).

5 Véanse exposiciones sobre la importancia de las capacidades de innovación tecnológica de los sectores en Kim y Nelson (2000), Nelson (1996), Rasgotra (2013), Taylor (2016) y Shin y Chang (2003).

6 Véanse análisis sobre la selección de sectores en Felipe (2015) y Falck, Gollier y Woessmann (2011).

7 La complementariedad entre la educación y otros factores se considera la fuerza que impulsa el crecimiento económico y la eficiencia en Asia oriental. Los sistemas de educación de Asia oriental se forman y se amplían en estrecha relación con las etapas de desarrollo económico: cuanto mayor es el nivel de desarrollo económico, mayor es la demanda de un mejor sistema de educación superior (Permani, 2009). 
El modelo supone un Gobierno fuerte y legítimo, un nacionalismo sólido, una burocracia eficiente y el control por parte del Gobierno del sistema financiero, lo que le permite canalizar recursos hacia los sectores estratégicos por mandato. Con el MEGA es posible analizar los efectos de diferentes políticas comerciales (aranceles o subsidios a ciertos tipos de importaciones, impuestos o subsidios a ciertas exportaciones), de modificaciones del tipo de cambio o de la utilización de impuestos o subsidios para limitar o expandir el consumo global (para reducir o aumentar el ahorro privado) o de cierto tipo de productos. De la misma manera, el MEGA puede simular restricciones en la entrada de inversión extranjera agregada y podría modificarse para analizar los efectos de una regulación selectiva de la inversión extranjera por industria.

Para simplificar y al mismo tiempo ilustrar las posibilidades del modelo desarrollador, se utiliza el MEGA, que permite analizar los efectos solo de dos políticas. Concretamente, se toman como supuestos: i) el otorgamiento de subsidios a la utilización de insumos nacionales para los sectores considerados claves, pero no para el resto de los sectores, y ii) que es posible motivar a la población para que incremente el ahorro, no por medio de impuestos al consumo - como lo hicieron en su momento la República de Corea (Liang, 2010) o Singapur (Huff, 1995) - y que el Gobierno puede canalizar estos mayores ahorros hacia los sectores claves (aquellos que presentan efectos de "arrastre" y "dispersión" superiores al promedio) ${ }^{8}$.

Las medidas proteccionistas y las de promoción de exportaciones no son mutuamente excluyentes. El modelo de crecimiento asiático ha sido presentado como un modelo en que el crecimiento se logra a través de las exportaciones, en contraposición al latinoamericano, de sustitución de importaciones, infructuoso. La equivocada dicotomía planteada al respecto dibujaba una América Latina proteccionista e ineficiente, frente a economías asiáticas que abrían sus fronteras a la competencia internacional y se beneficiaban de una creciente demanda internacional a través del comercio basado en sus ventajas comparativas. Si se analizan los casos exitosos del Japón en los siglos XIX y XX, de la República de Corea y la provincia china de Taiwán en el siglo XX y, más recientemente, de China y Viet Nam, y el papel que desempeñaron el comercio exterior o la inversión extranjera directa (IED), se observa que no es una relación lineal.

Los respectivos Gobiernos de esos países ofrecieron en su momento interesantes incentivos, como créditos preferenciales, licencias que limitaban la competencia interna y externa, y aranceles o acceso a divisas con ventajosos precios oficiales, pero al mismo tiempo exigían metas de exportación para reducir los déficits en cuenta corriente. La exigencia de metas de exportación tendría, con el tiempo, un beneficio superior al de equilibrar el sector externo. Las metas obligaban a los empresarios de esos países a lograr un buen desempeño en un mercado que no estaba intervenido y, en consecuencia, en el que no contaban con privilegios, al contrario de lo que sucedía en el mercado interno. La obtención de beneficios en un mercado en el que, a través de licencias, se eliminaba la posibilidad de competencia no inducía a un tejido productivo ineficiente; por el contrario, los conglomerados alemanes (karteles), los japoneses (zaibatsu), los coreanos (chaebol) o los chinos podían disminuir artificialmente los precios de exportación, por debajo de los propios costos, a fin de lograr la meta determinada de exportaciones y compensar dichas pérdidas con los beneficios extraordinarios que obtenían en su mercado interno. El mercado exterior permitió y permite así corregir los peligros locales de los incentivos de la políica industrial (Berasaluce y Romero, 2018).

Para el MEGA que proponemos se toma como supuesto que en algunos sectores existen rendimientos crecientes a escala. Estos rendimientos crecientes son internos de la industria, pero externos de la empresa, lo que permite conservar el supuesto de competencia perfecta (Helpman y Krugman, 1985). Debido a que trabajamos con rendimientos crecientes, el modelo no llega a un estado estable. El número de períodos es 10 y el MEGA cuenta con 47 sectores.

\footnotetext{
8 EI MEGA puede perfectamente simular este objetivo mediante impuestos al consumo, pero consideramos que suponer el aumento del ahorro como medida voluntaria, tomada por razones no económicas, inducida por un sentimiento nacional, que va más allá del individualismo y tiene la perspectiva de lograr una nación fuerte y poderosa, es más congruente con el funcionamiento del modelo desarrollador.
} 
El presente trabajo se organiza como sigue: en la segunda sección se detalla y fundamenta la función de rendimientos crecientes a escala que se utiliza; en la tercera sección se describe la base de datos, la construcción de la matriz de contabilidad social y la selección de los sectores claves; en la cuarta se describe el MEGA propuesto (véase el modelo matemático en el anexo A3); en la quinta se implementa la simulación propuesta y se analizan los resultados y, finalmente, en la sexta sección, se concluye con algunos comentarios y reflexiones finales.

\section{Rendimientos crecientes ${ }^{9}$}

Aunque los rendimientos crecientes a escala pueden obedecer a varias causas, el papel principal tradicionalmente se ha atribuido a la división del trabajo. De acuerdo con Sánchez (2011), las ideas de Adam Smith sobre este tema fueron posteriormente mejoradas por Young (2009) y después indirectamente por una serie de economistas del desarrollo -Rosenstein-Rodan (1959), Nurkse (1963), Hirschman (1961), Myrdal (1959) y Prebisch (1960), entre otros - para quienes la industria constituía el motor del crecimiento, ya fuera por los rendimientos crecientes a escala o por sus encadenamientos productivos.

Más recientemente, otros autores han realizado adiciones conceptuales importantes, entre las que destacan los trabajos de Krugman (The Nobel Prize, 2020) y Helpman y Krugman (1985), en los que se fundamenta el concepto de rendimientos crecientes a escala empleado en la presente investigación.

En el terreno empírico se ha desarrollado a nivel internacional un ingente acervo de trabajos dedicados a probar la hipótesis de rendimientos crecientes a escala en economías reales y a estimar parámetros que dan razón de su cuantía. Por ejemplo, Antweiler y Trefler (2000) encontraron que el marco Helpman-Krugman aportaba un notable lente para ver las elasticidades de equilibrio general a escala codificadas en los flujos de comercio y, en particular, que un tercio de todas las industrias productoras de bienes se caracterizaban por su escala (el rango modal de las elasticidades a escala para este grupo era de 1,10-1,20 y la elasticidad de la economía a gran escala era de 1,05).

Para el caso específico de México, también se han realizado estudios que demuestran que estamos ante efectos significativos. Castañeda y Garduño (2000) estiman un índice de rendimientos a escala para las industrias manufactureras mexicanas, en un trabajo en que se encuentra "bastante evidencia de rendimientos crecientes a escala" (pág. 279), y concluyen que, considerando los resultados de los datos agrupados, "seis sectores que incorporan 21 industrias tienen evidencia de rendimientos crecientes a escala. En las estimaciones a nivel de dos dígitos, 19 industrias muestran rendimientos crecientes a escala" (pág. 296).

Sánchez (2011) analiza el estancamiento en México en su relación con las manufacturas y los rendimientos crecientes desde un enfoque kaldoriano. A partir de una serie de modelos econométricos, somete a prueba tres leyes de Kaldor y encuentra, entre otros resultados, que "la estimación de la ley con la especificación de Kaldor indica que el grado de rendimientos crecientes es de alrededor de 2.98 en las regiones mexicanas, la prueba de Wald indica que este valor es estadísticamente significativo".

Por otra parte, también se han utilizado los rendimientos crecientes a escala en trabajos teóricos aplicados para demostrar implicaciones empíricas en modelos de equilibrio general aplicado, como lo hacen López-de-Silanes, Markusen y Rutherford (1992) con el caso de México.

En concordancia con los trabajos antes referidos para esta investigación, a partir de la propuesta de Helpman y Krugman (1985) de una función de producción con rendimientos crecientes a escala $Y=g(Y) \tilde{f}(v)$, donde $v$ es el vector de insumos, planteamos una función de rendimientos crecientes a

9 Esta sección se basa en Young (2009). 
escala con la forma funcional específica $g=\left(\frac{Y_{t}}{Y_{t-1}}\right)^{\mu}$, donde $Y_{t}$ es el nivel de producción actual e $Y_{t-1}$ el anterior; $\mu$ puede especificarse a distintos niveles para lograr grados más o menos altos de rendimientos crecientes a escala; en la simulación principal especificamos $\mu=1,5$ para 12 sectores claves, lo que es congruente con los resultados de Sánchez (2011) y de Castañeda y Garduño (2000) para México. Para los restantes 35 sectores especificamos $\mu=0$ (es decir, no desarrollan rendimientos crecientes a escala). Es preciso notar que se trata de un supuesto muy conservador, pues con el crecimiento de la economía también se generarían rendimientos crecientes, en algún grado, en los sectores que no son claves; en simulaciones con una $\mu$ mayor para cualquiera de los dos grupos (o para ambos), como es de esperarse, los resultados que se obtienen son siempre superiores a medida que se incrementa $\mu$.

\section{Datos y matriz de contabilidad social}

El flujo circular de la economía es el concepto sobre el que se construye una matriz de contabilidad social, en donde es posible incluir todos los flujos de la economía con mayor o menor desagregación; a su vez, la matriz de contabilidad social constituye la base de datos estándar sobre la que se diseña un MEGA.

Los hogares poseen los factores productivos, que son contratados por las empresas para producir bienes y servicios que venden a los hogares. Se supone que la economía, en cada ciclo productivo, alcanza un equilibrio que permite a los agentes optimizar sus funciones de comportamiento, y que los mercados de factores y de bienes se vacían.

El Gobierno recauda impuestos de los hogares y las empresas para aportar servicios y, finalmente, la economía del país intercambia bienes con el resto del mundo.

El flujo circular de la economía genera, a su vez, un flujo circular del ingreso con un contraflujo también circular de bienes y factores. Cuando las empresas contratan capital y trabajo generan un ingreso para los hogares, que lo usan para comprar los bienes producidos; las empresas a su vez utilizan esos ingresos para volver a contratar capital y trabajo, y así sucesivamente.

Para elaborar la matriz de contabilidad social que constituye la base de datos para el diseño, calibración e implementación del modelo, recurrimos como punto de partida a la matriz de insumo-producto simétrica nacional publicada por el INEGI para 2012 (INEGl, 2016) (agregada a 47 actividades productivas) y a datos de las cuentas nacionales para cerrar las cuentas. Adicionalmente, empleamos la matriz de importaciones por origen y destino (INEGl, 2016), una característica novedosa de nuestro modelo, que permite detallar minuciosamente la combinación CES (elasticidad de sustitución constante (constant elasticity of substitution)) de insumos nacionales y extranjeros que se utiliza en cada caso (insumos compuestos).

Para definir los sectores beneficiados por la política activa de subsidio a insumos nacionales (con el efecto de incrementar el contenido nacional), utilizamos el análisis de sectores claves basado en los índices de Rasmussen (1963)10 (véase el anexo A1). Los sectores seleccionados son 12: energía eléctrica; industria alimentaria; industria del papel; fabricación de productos derivados del petróleo y del carbón; industria química; industria del plástico y del hule; industrias metálicas básicas; fabricación de productos metálicos; fabricación de maquinaria y equipo; fabricación de equipo de computación, comunicación y otros; fabricación de accesorios, aparatos y equipos eléctricos; y fabricación de equipo de transporte ${ }^{11}$.

\footnotetext{
${ }^{10}$ Los índices de Rasmussen (1963) consisten en calcular las magnitudes medias de los multiplicadores de arrastre y de dispersión para cada uno de los sectores productivos y compararlos con la media global, a fin de identificar los "sectores claves", que son aquellos cuyos multiplicadores, tanto de arrastre como de dispersión, están por encima de la media. En esta investigación los utilizamos para definir los sectores que perciben los beneficios de una política pública específica y, a la vez, para especificar los sectores con rendimientos crecientes a escala.

11 Véase un estudio con resultados positivos de economías de escala en México en Castañeda y Garduño (2000).
} 


\section{Modelo dinámico-recursivo de equilibrio general aplicado (MEGA)}

En este apartado se hace una descripción intuitiva del modelo. En el anexo A2 se incluye la lista de parámetros y variables que sirven como referencia para la subsecuente especificación del modelo matemático. En el cuadro A2.1 del anexo A2 se describen los parámetros a calibrar con los datos de la economía contenidos en la matriz de contabilidad social. Del mismo modo, en el cuadro A2.2 se describen las variables del modelo. En el anexo A3 se especifica el modelo matemático.

\section{Hogares}

En la matriz de contabilidad social-Mx12 los hogares se encuentran agregados en una cuenta que concentra todos los ingresos privados en la primera fila de la matriz y todos los gastos en la primera columna. En la economía representada por la matriz de contabilidad social los hogares obtienen ingresos por rentas de capital, por sueldos y salarios, transferencias gubernamentales y transferencias (remesas) del resto del mundo (RdM).

Por otra parte, los hogares pagan impuestos y el resto de su ingreso disponible lo destinan al ahorro (consumo futuro) y al consumo de bienes y servicios (consumo presente). A su vez, el consumo de bienes y servicios se descompone en dos, un bien agregado nacional y un bien agregado importado.

\section{Gobierno}

El Gobierno recauda impuestos: sobre la renta, sobre los productos y sobre la producción. Con estos, realiza transferencias a los hogares (programas de combate a la pobreza y otros), ahorra una parte y el resto lo dedica a la compra de bienes nacionales e importados.

\section{Ahorro e inversión}

La cuenta de ahorro e inversión primero concentra el ahorro de los agentes: hogares, empresas, Gobierno y resto del mundo (cuenta corriente de la balanza de pagos, que equivale al préstamo neto del resto del mundo), para financiar la compra de bienes de capital (formación bruta de capital fijo, incluida la variación de existencias). Una parte de los bienes de capital proviene de la oferta total nacional y una parte del resto del mundo.

\section{Factores productivos}

La economía cuenta con una dotación inicial de capital indiferenciado y otra de trabajo, también uniforme. Esto implica plena movilidad de factores entre las actividades productivas, lo que a su vez supone un horizonte temporal de largo plazo, es decir, eventualmente el capital puede ser transformado y trasladado de unas actividades a otras en función de los estímulos generados por cambios en los precios relativos. Lo mismo se aplica al trabajo.

\section{Producción}

La producción de la oferta total de bienes y servicios en la economía se modela a través del siguiente anidamiento: primero se produce el valor agregado a través de una función Cobb-Douglas; al mismo 
nivel se genera un insumo compuesto (insumos nacionales e insumos importados) a través de una función CES; en el siguiente nivel se lleva a cabo la estimación de la producción de la oferta total con una función Leontief.

Dada su complejidad, el anidamiento de la producción en los modelos de equilibrio general aplicado es una práctica estándar para su diseño e implementación, pues de otro modo sería inmanejable tanto para la especificación matemática de los modelos como para su implementación computacional (véase, por ejemplo, Lofgren, Harris y Robinson, 2002).

\section{Resto del mundo (RdM)}

El resto del mundo obtiene ingresos de la importación de insumos y de bienes de capital, así como de las importaciones directas de los sectores público y privado, y también recibe una parte de la renta de capital, principalmente como pago de rentas a la propiedad. Los gastos en que incurre el resto del mundo son: transferencias a los hogares (remesas), pago al trabajo (documentado), exportaciones y ahorro (préstamo neto del resto del mundo).

\section{Cierres macroeconómicos}

Los cierres macroeconómicos especificados para implementar las simulaciones son:

i) propensión marginal fija al ahorro e inversión variable;

ii) tasas impositivas fijas y recaudación variable, y

iii) tipo de cambio fijo e ingreso del resto del mundo variable

\section{Dinámica recursiva}

Dixon y Parmenter (1996) distinguen cuatro casos de modelación dinámica. En este modelo seguimos el primero, en que la inversión es exógena, congruente con expectativas de alcance limitado, las tasas de ahorro están dadas y el ahorro es igual a la inversión en cada período. La población (trabajo) crece a una tasa constante $g_{0}$. Para la dinámica recursiva utilizamos proyecciones externas del crecimiento de la población económicamente activa (PEA), en promedio del 1,6\% por período (Partida, 2008).

\section{Simulaciones y resultados}

Simulamos una política de impulso al crecimiento basada en dos elementos: un incremento en la propensión marginal al ahorro de los hogares como resultado de la persuasión moral (como lo realizado en la República de Corea con el eslogan "gasta lo que te sobre después de ahorrar" (Park, 1979)) o bien mediante impuestos directos al consumo y subsidios al uso de insumos nacionales. Como ya se mencionó, se toma como supuesto que 12 sectores claves presentan rendimientos crecientes a escala tanto en el escenario base (o de referencia) como en las simulaciones.

\section{Escenario base}

Para obtener el escenario base en un horizonte de diez períodos, se parte de la tasa de acumulación de capital observada en el año cero (equilibrio inicial) y, como ya se indicó, de un crecimiento del factor trabajo, según las proyecciones de Partida (2008), del 1,6\% en promedio. 
Las matrices insumo-producto publicadas por el INEGI (2016), que es la fuente que se utilizó, incluyen todas las remuneraciones pagadas en la economía, incluso las del sector informal. Para los propósitos de este análisis no es relevante la desagregación de las remuneraciones según sector formal e informal; es preciso notar, de todas formas, que en el sector manufacturero prácticamente la totalidad del trabajo es formal (véase el cuadro 1).

\section{Cuadro 1}

Composición del valor agregado total y del factor trabajo

(En millones de pesos constantes de 2012)

\begin{tabular}{lr}
\hline Valor agregado bruto (precios básicos) & 15106358,629 \\
\hline Total de remuneración de los asalariados & 4216575,358 \\
\hline Salarios & 782354,771 \\
\hline Sueldos & 3010643,774 \\
\hline Contribuciones sociales efectivas a la seguridad social & 317929,494 \\
\hline Otras prestaciones sociales & 105647,319 \\
\hline Impuestos netos sobre la producción & 84631,434 \\
\hline Excedente bruto de operación & 10805151,837 \\
\hline
\end{tabular}

Fuente: Instituto Nacional de Estadística y Geografía (INEGI), "Sistema de cuentas nacionales de México: fuentes y metodología, año base 2013", 2017 [en línea] https://www.inegi.org.mx/contenidos/programas/mip/2013/metodologias/SCNM_ Metodo_MIP_B2013.pdf.

\section{Simulaciones}

Una vez obtenidos los resultados del escenario base, se implementó la siguiente simulación combinada: un incremento del 10\% al 20\% en la propensión marginal al ahorro de los hogares, y un subsidio del $20 \%$ a la compra de insumos nacionales para los sectores claves ${ }^{12}$.

Los subsidios son costeados por la recaudación gubernamental. Lógicamente, al tener que dedicar una magnitud creciente a este subsidio (dado que se especifica un 20\% para una economía cada vez mayor), la recaudación y, por tanto, los gastos del Gobierno decrecen. Pero esto se compensa con creces por el aumento del consumo real de los hogares y por el incremento de su ingreso, al crecer los acervos de capital, dado que el ahorro es creciente.

En los gráficos 1 y 2 se presentan los resultados para la productividad del trabajo en dos grupos de sectores. En el gráfico 1, los sectores electricidad, alimentos y derivados del petróleo y el carbón reaccionan poco ante el aumento del ahorro y el subsidio a los precios de los insumos nacionales; en contraste, los sectores industria del papel, industria química e industria del plástico y del hule presentan un incremento de la productividad de entre un $84 \%$ y un $98 \%$ por encima del que se lograría en caso de no aplicarse política alguna.

Aunque las dos políticas aquí consideradas son relativamente sencillas, los efectos en paralelo y en serie que se desencadenan al aplicarlas son muy complejos debido a las numerosas interrelaciones que tienen lugar entre los sectores productivos y entre estos y el resto de los agentes económicos, así como a todos los efectos de segundas y subsecuentes vueltas. En general, la evolución de una industria particular, así como de su productividad, dependerá de varios factores, principalmente de la magnitud de los multiplicadores de arrastre (en este caso, por el subsidio a los insumos nacionales), de su importancia creciente como proveedora de insumos para las demás industrias y de la relación entre capital y trabajo que mantiene.

\footnotetext{
${ }^{12}$ Además de las economías de escala, el residuo de Solow también puede ser influido por incrementos en la productividad conjunta de los factores y por la introducción de mejoras tecnológicas.
} 


\section{Gráfico 1}

Comparación del incremento de la productividad del trabajo: escenario base respecto de escenario con aplicación de políticas, sectores de baja respuesta (Índice, año base 2017 = 1)

A. Energía eléctrica

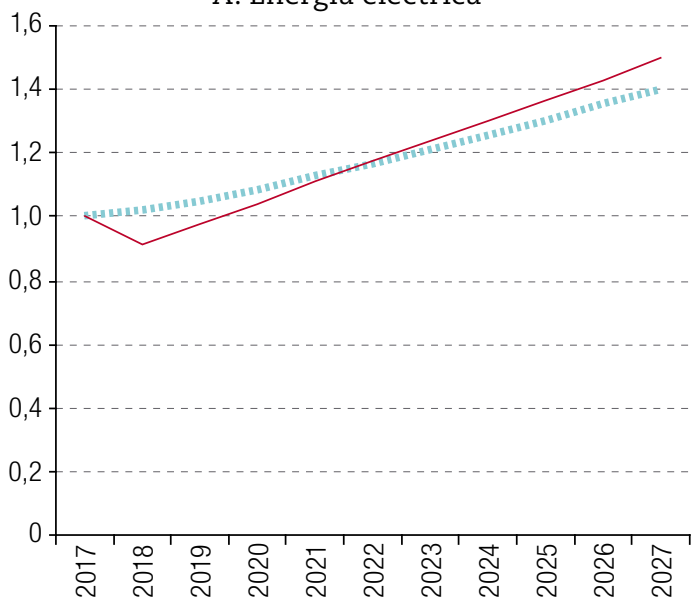

C. Industria del papel

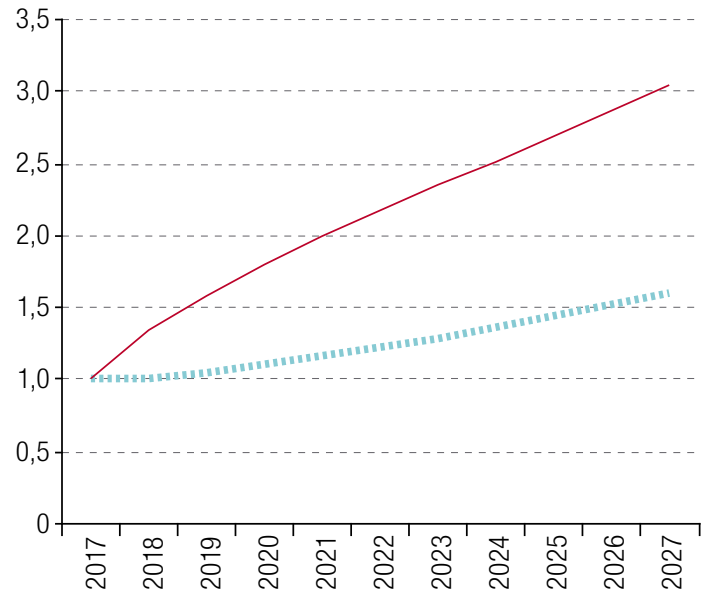

E. Industria química

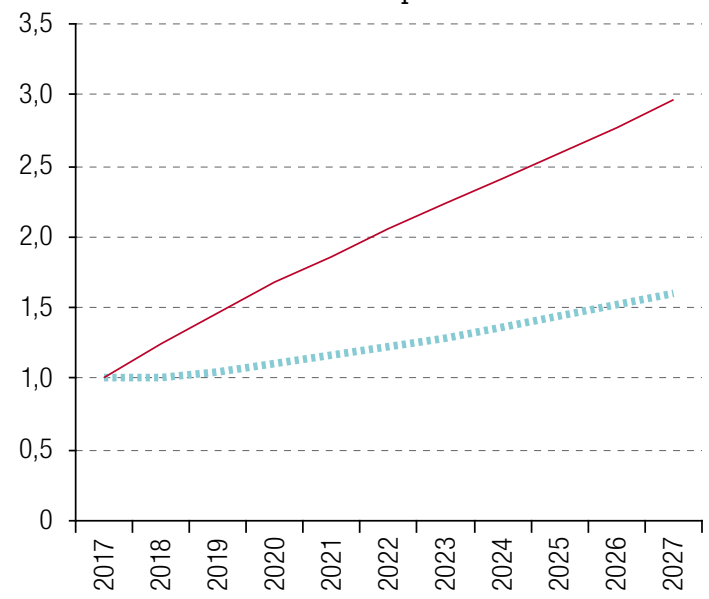

" и. Escenario base
B. Industria alimentaria

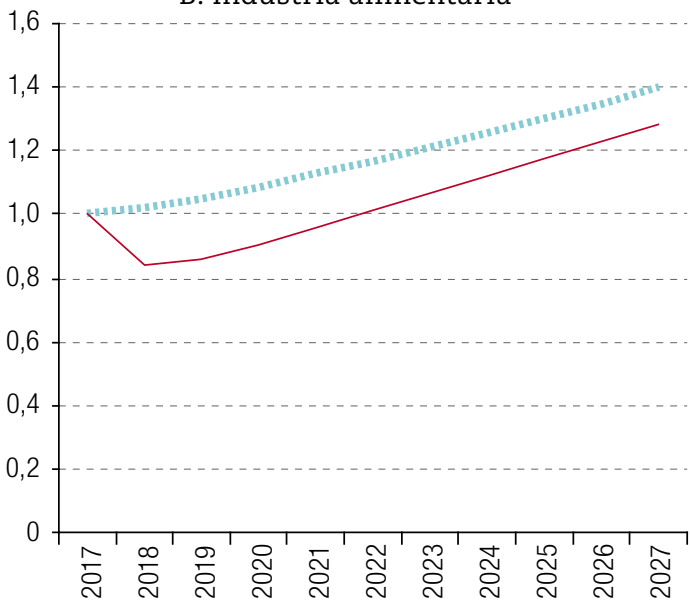

D. Derivados del petróleo y del carbón

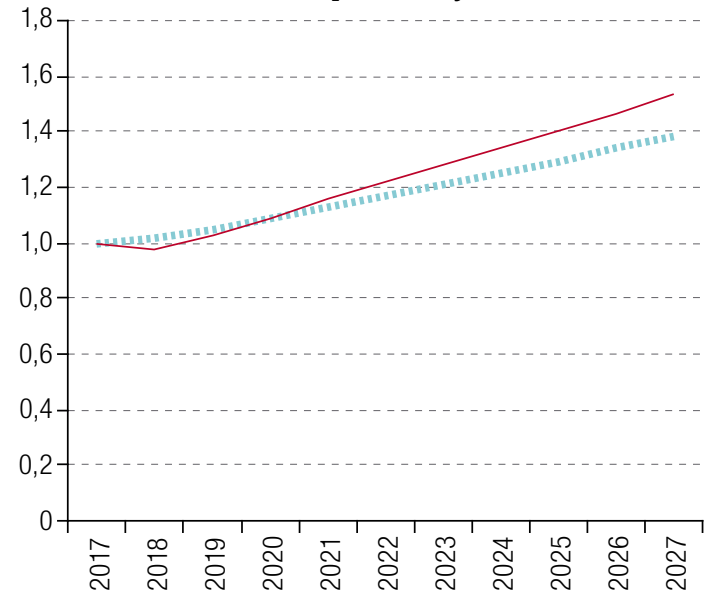

F. Industria del plástico y hule

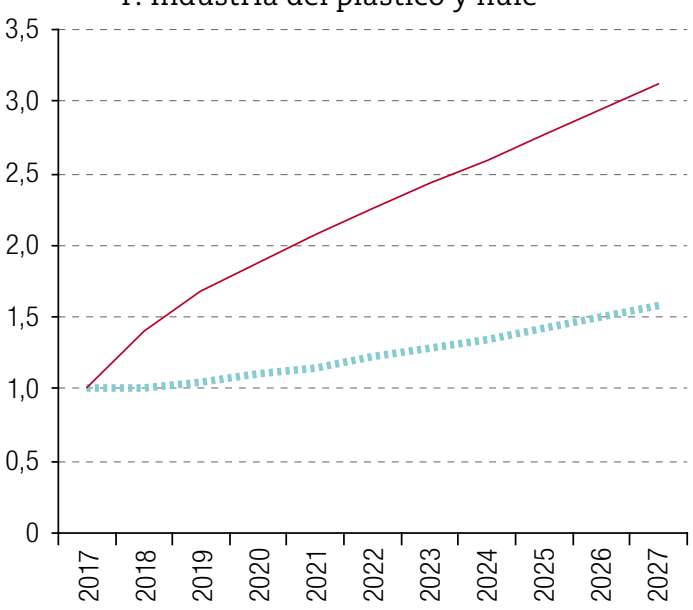

Simulación con la aplicación de políticas

Fuente: Elaboración propia. 
En el gráfico 2 se presenta el segundo grupo favorecido. En este caso, en las industrias metálicas básicas y la fabricación de productos metálicos, maquinaria y equipo, equipo de computación, comunicación y otros, aparatos eléctricos, y equipo de transporte (industria automotriz), el incremento de la productividad es notable. El sector que presenta el menor crecimiento de la productividad es el sector de equipo de transporte, con un 52\%, y el que presenta el mayor incremento es el de industrias metálicas básicas, que triplica su productividad en solo diez años.

En la economía en su conjunto también se producen efectos positivos. La productividad total del trabajo en el décimo período llega a ser un 7\% superior a la del escenario base. En el gráfico 3 se presenta la evolución indizada de la productividad del trabajo (período $0=1$ ), agrupando las actividades productivas en seis grandes sectores: i) agropecuario, ii) petróleo y minería, iii) electricidad y gas, iv) construcción, v) manufacturas y vi) servicios (véase el anexo A4).

En un escenario con aplicación de políticas, el sector agropecuario incrementa su productividad en un $2 \%$ para el décimo año con respecto al escenario base, el sector petróleo lo hace en un 26,56\%, electricidad y gas solo en un 3,63\%, construcción en un 39,8\% y manufactura en un 37,14\%, en tanto que en el sector servicios la productividad en el décimo año es un 4,3\% menor que en el escenario base.

Estos movimientos permiten que la participación del sector manufacturero en el PIB en el décimo año pase del 19,6\% en el escenario base al 21,95\% en el escenario con la aplicación de las políticas, es decir, que presente un incremento de 2,35 puntos porcentuales en solo diez años (véase el gráfico 4).

Como señala Coatsworth (1990), el ingreso por habitante, a pesar de sus limitaciones, constituye el mejor indicador del bienestar; si con una política económica se incrementa el ingreso por habitante de un país, esto indica que potencialmente está mejor que antes. Mediante la "prueba de la compensación" se puede suponer que, si el ingreso por habitante aumenta, los ganadores podrían compensar a los posibles perdedores y conservar un remanente. El ingreso por habitante de un país está estrechamente relacionado con la productividad promedio del trabajo en la economía ${ }^{13}$.

Por ello, para saber qué pasa con el ingreso por habitante es necesario conocer el comportamiento de la productividad del trabajo. En el gráfico 5 se muestran los resultados para la productividad del trabajo.

Utilizando datos de los anexos estadísticos de los Informes de Gobierno correspondientes a varios años, sobre PIB, empleo y población, se proyectó la trayectoria de la productividad del trabajo y del PIB por habitante para el período comprendido entre 1988 y 2027 (véase el gráfico 6).

Para verlo en perspectiva, la productividad del trabajo entre 1988 y 2017 solo se incrementó un 19\%, mientras que con las políticas sugeridas en diez años se lograría un aumento de la productividad del 18\%. Visto de otra forma, con las reformas sugeridas, la productividad del trabajo en diez años más sería un 18\% superior a la de 2017, en lugar de un 10\%, que sería el incremento si no se aplican dichas reformas.

Por su parte, en el escenario base, el ingreso por habitante registraría una tasa de crecimiento del 0,95\% en el mismo período, en tanto que con las políticas sugeridas dicha tasa sería del 1,67\%. Este resultado por sí solo justificaría adoptar una estrategia de crecimiento liderada por el Estado. Esta tasa de incremento del PIB por habitante no se logró ni siquiera entre 1988 y 2017, cuando fue del 1,27\%, resultado que no se debió a incrementos de la productividad (que solo creció durante ese período a una tasa del 0,61\%), sino a un notable crecimiento de la proporción de la población incorporada al mercado de trabajo, del 0,66\% (es decir, la diferencia entre $1,27 \%$ y $0,61 \%)^{14}$ (véase el gráfico 7 ).

\footnotetext{
${ }^{13}$ El PIB por habitante $(P I B / P)$ puede ser descompuesto en productividad media del trabajo (PIB/E), tasa de participación de la población en la fuerza de trabajo $(L / P)$ y tasa de empleo $(E / L)$, donde PIB: producto interno bruto; P: población; L: fuerza de trabajo, y E: empleo. Esto es: $\frac{P I B}{P}=\left(\frac{P I B}{E}\right)\left(\frac{L}{P}\right)\left(\frac{E}{L}\right)$. Esta identidad muestra que las variaciones observadas en el $\mathrm{PIB}$ por habitante responden a factores relacionados con la productividad del trabajo, el nivel de actividad económica y factores relacionados con tendencias socioeconómicas.

${ }^{14}$ Suponiendo que hay pleno empleo, la tasa de crecimiento del PIB por habitante puede expresarse como la suma de la tasa de crecimiento de la productividad media del trabajo y la tasa de crecimiento de la participación de la población en el empleo: $\left(\frac{P I B}{P o b}\right)^{0}\left(\frac{P I B}{E m p}\right)^{0}+\left(\frac{E m p}{P O b}\right)^{0}$ donde el superíndice ${ }^{0}$ indica tasas de crecimiento.
} 


\section{Gráfico 2}

Comparación del incremento de la productividad del trabajo: escenario base respecto de escenario con aplicación de políticas, sectores de alta respuesta (Índice, año base $2017=1$ )

\section{A. Industrias metálicas básicas}

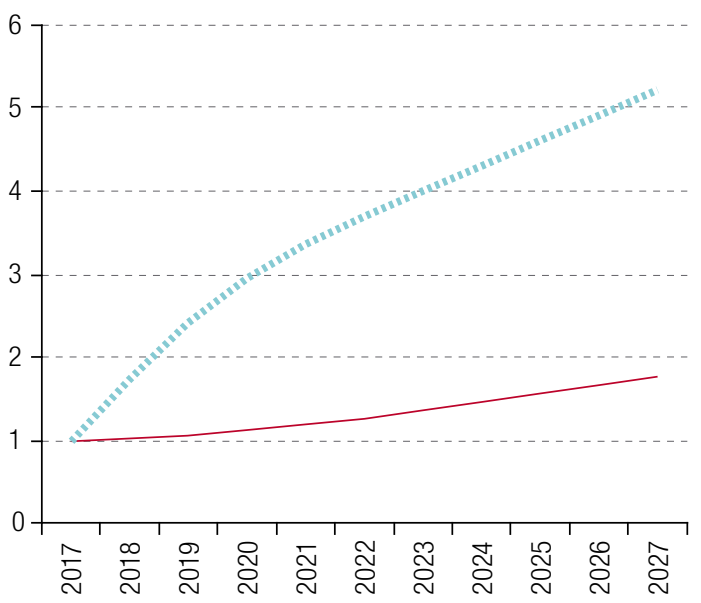

C. Maquinaria y equipo

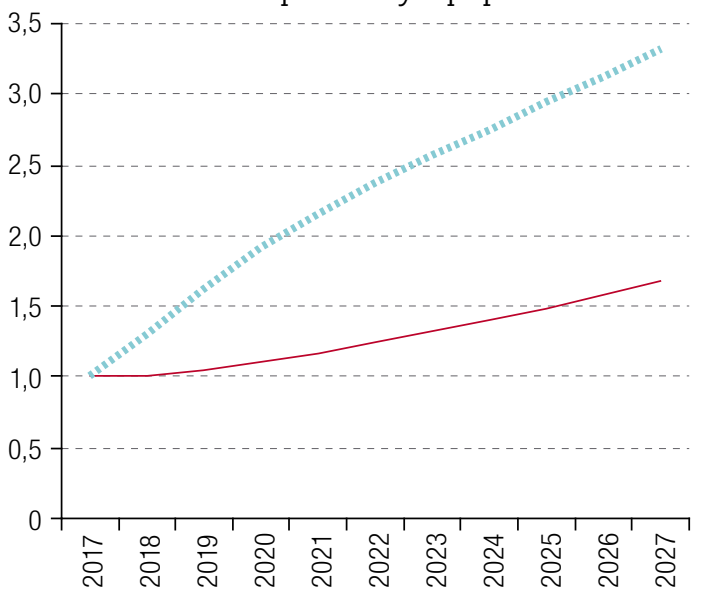

E. Aparatos eléctricos y equipo de generación de energía eléctrica

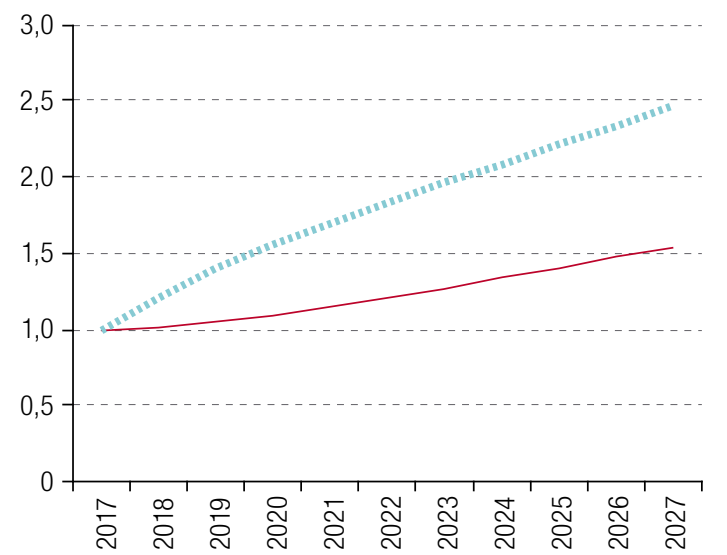

B. Productos metálicos

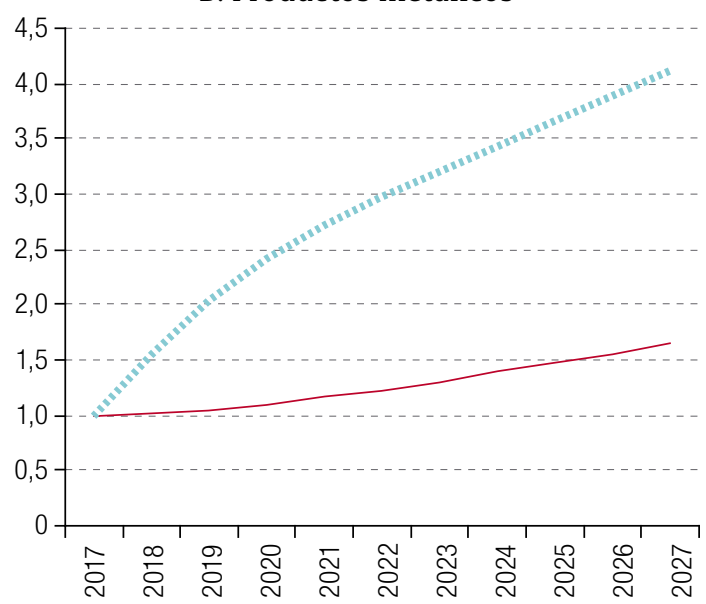

D. Equipo de computación, comunicación, medición y otros

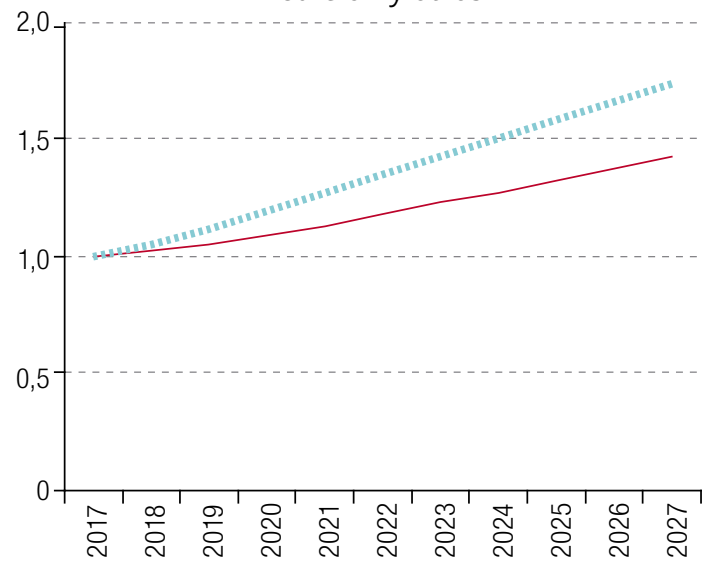

F. Equipo de transporte

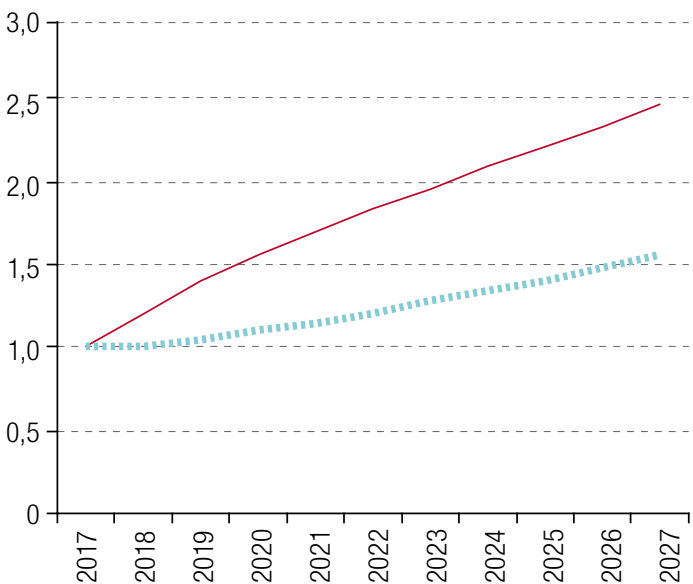

\#н. Escenario base — Simulación con la aplicación de políticas

Fuente: Elaboración propia. 


\section{Gráfico 3}

Índice de productividad por sector, escenario base y escenario con aplicación de políticas (Año base $2017=1$ )

A. Agricultura

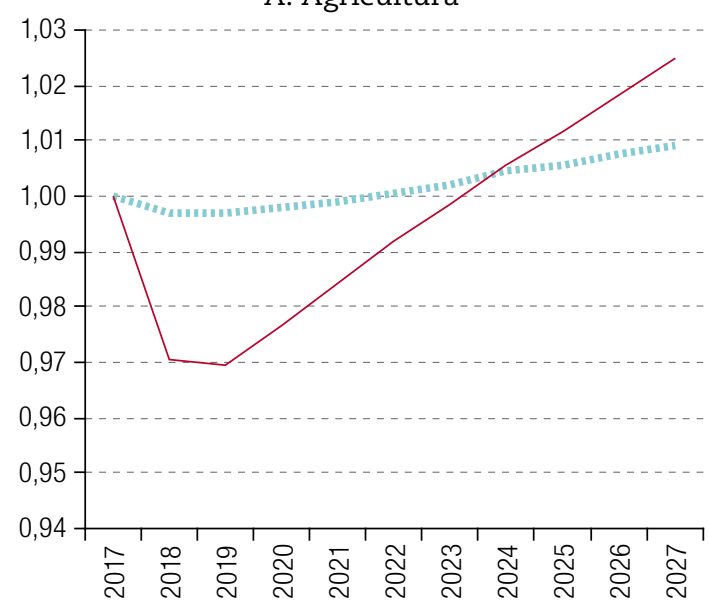

C. Electricidad y gas

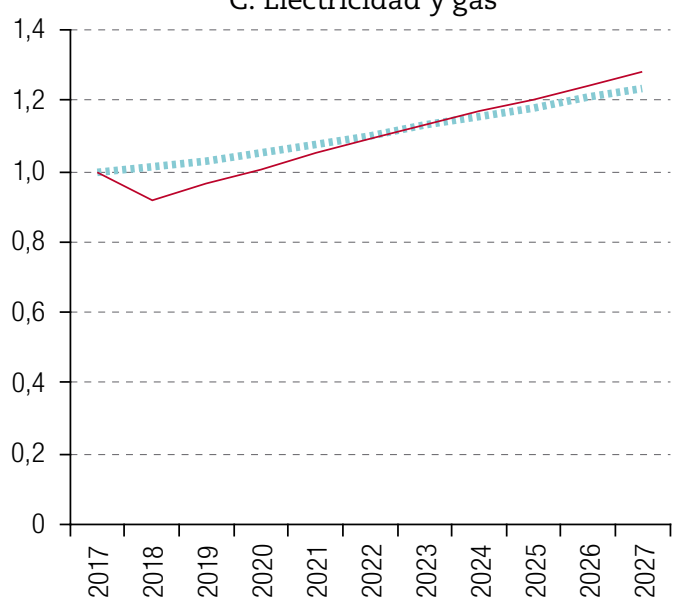

E. Manufacturas

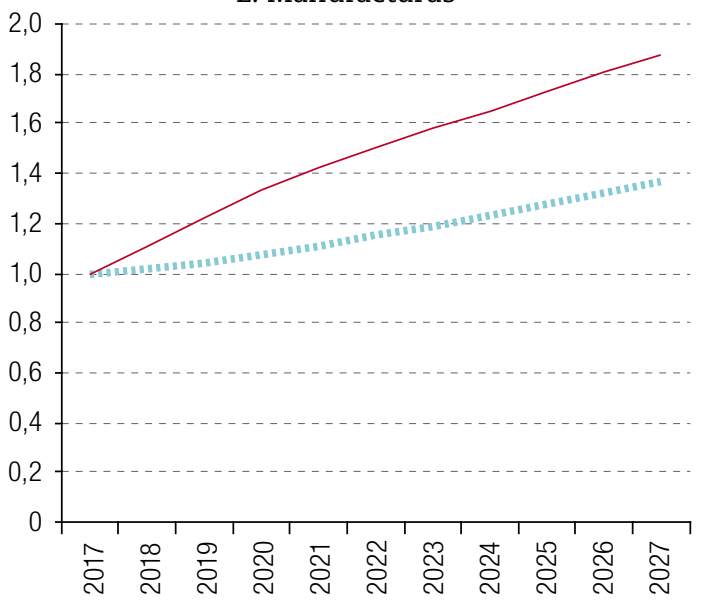

B. Petróleo y minería
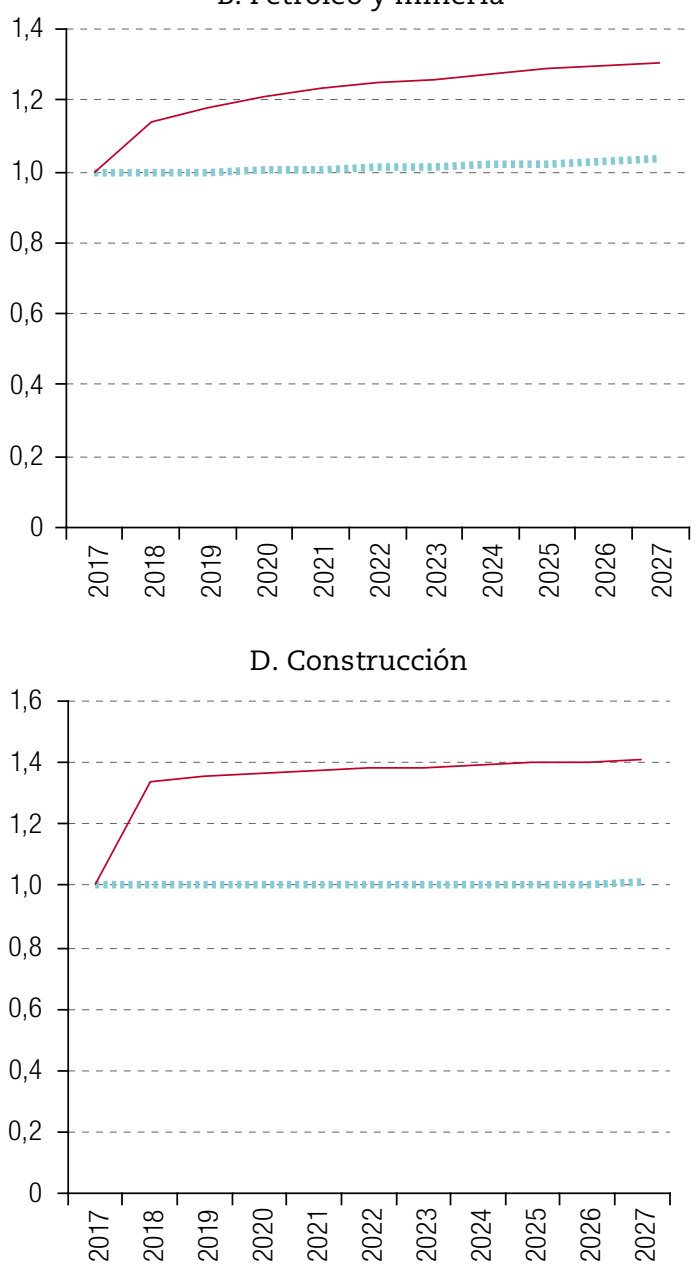

\section{F. Servicios}

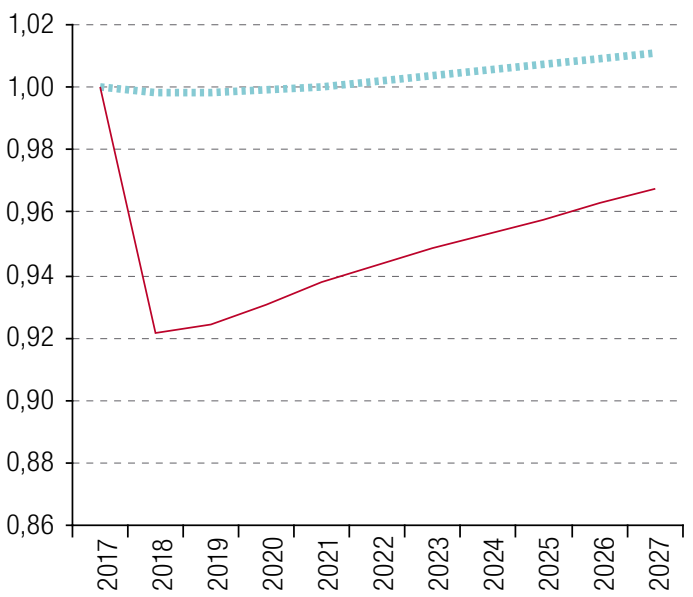

\#n.. Escenario base — Simulación con la aplicación de políticas

Fuente: Elaboración propia. 
Gráfico 4

Participación del sector manufacturero en el PIB nacional, escenario base y escenario con aplicación de políticas

(En porcentajes)

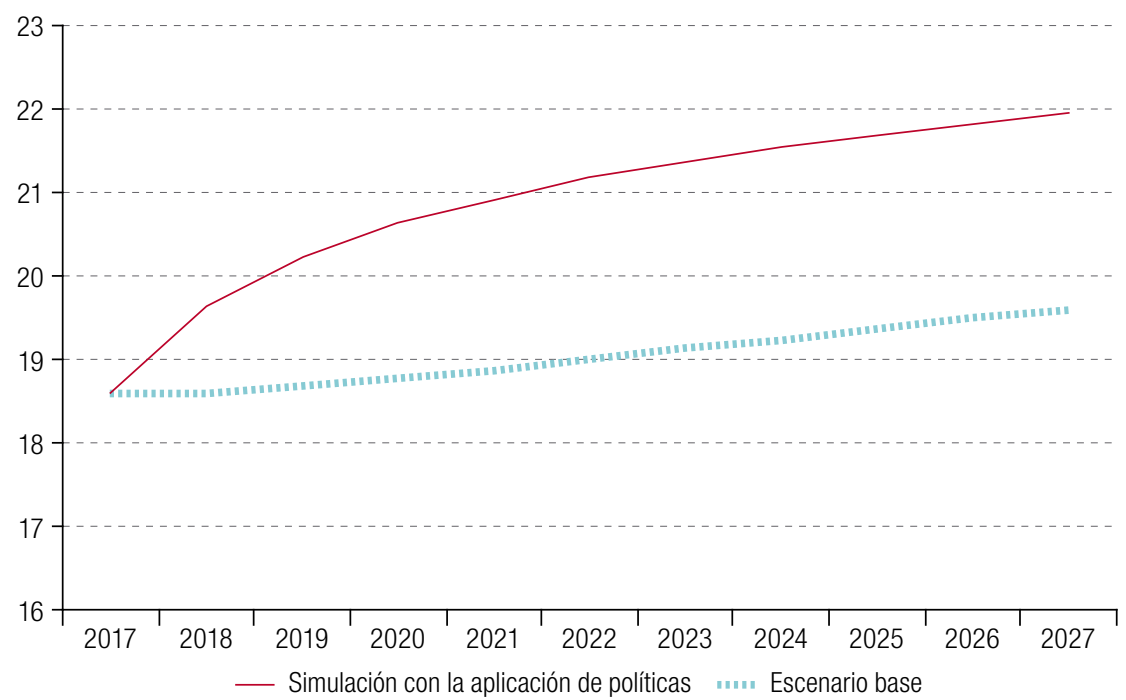

Fuente: Elaboración propia.

Gráfico 5

Índice de productividad del trabajo

(Año base $2017=1$ )

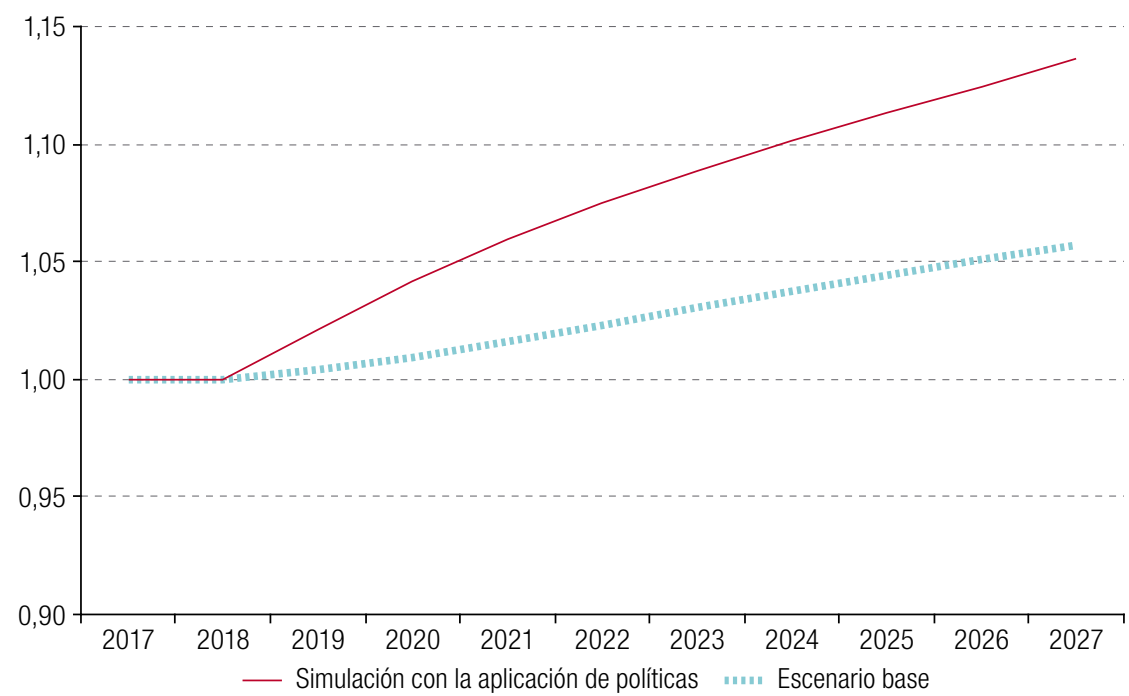

Fuente: Elaboración propia. 


\section{Gráfico 6}

Productividad del trabajo e ingreso por habitante, 1988-2027

(En miles de pesos constantes de 2008)

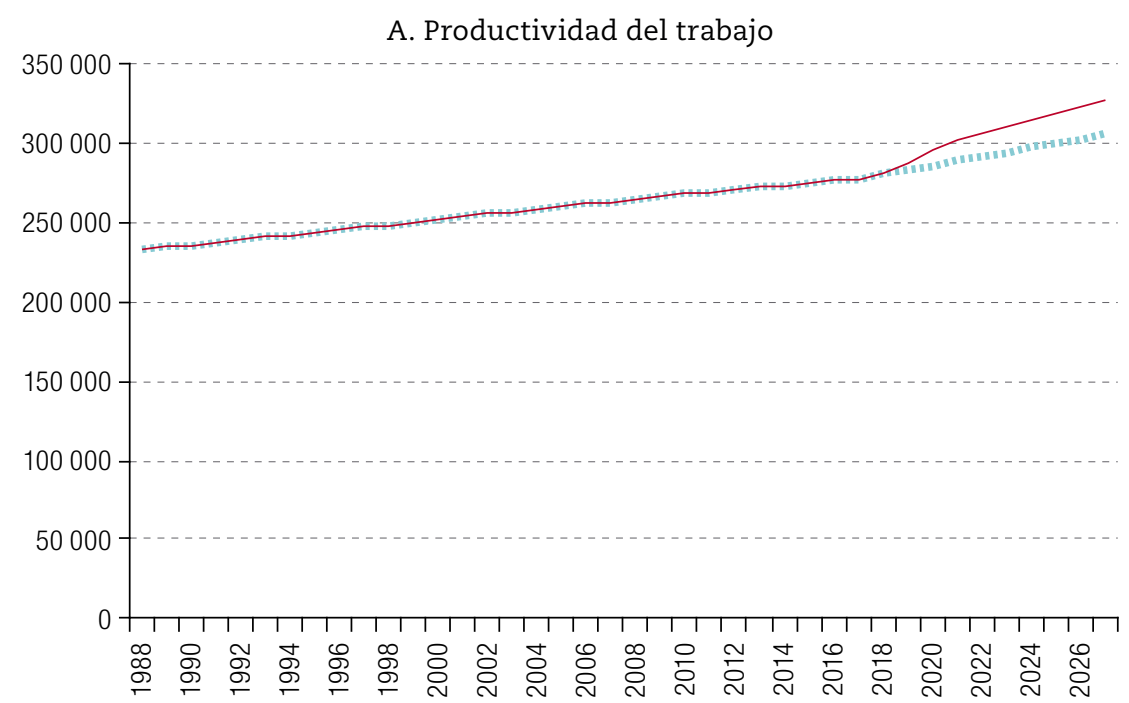

\section{B. Ingreso por habitante}

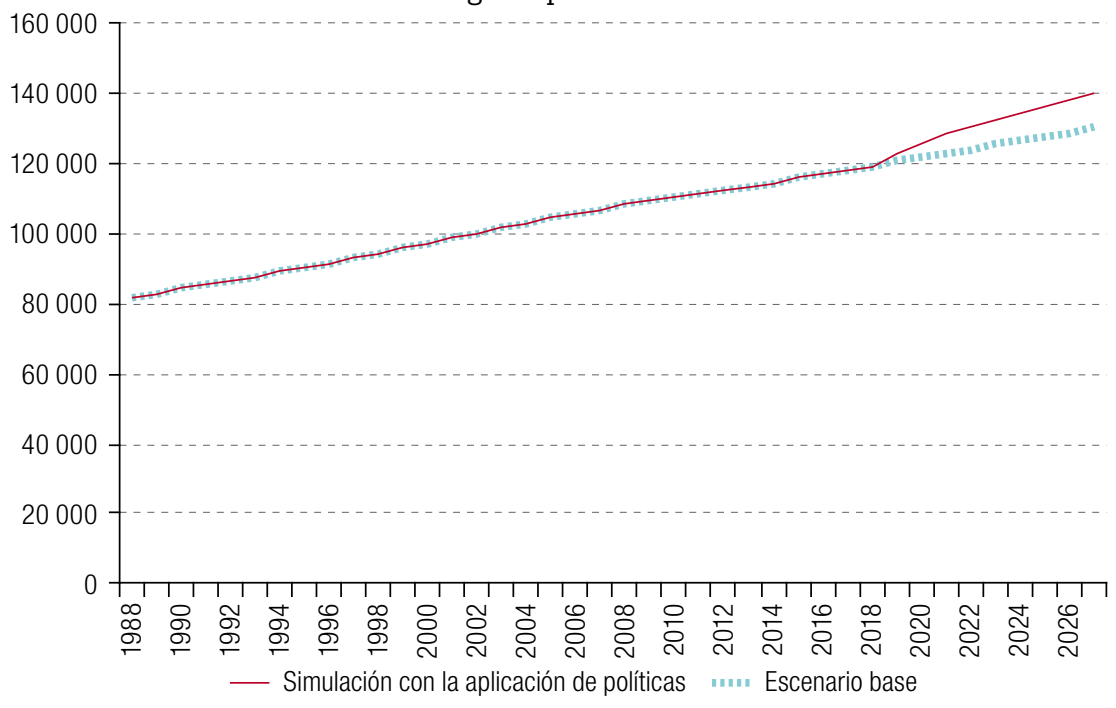

Fuente: Elaboración propia, sobre la base de México, Informe de Gobierno: anexo estadístico, Ciudad de México, varios años. 
Gráfico 7

Población ocupada como proporción de la población total, 1988-2017

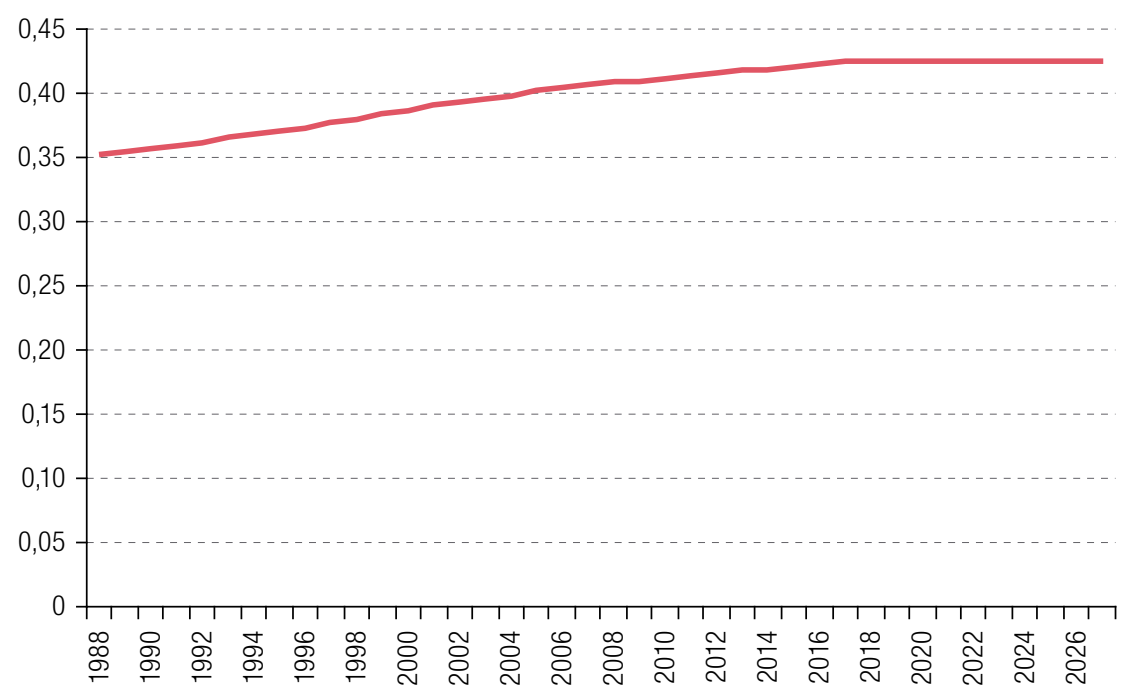

Fuente: México, $6 .{ }^{\circ}$ Informe de Gobierno: anexo estadístico, Ciudad de México, 2000; 5. Informe de Gobierno: anexo estadístico, Ciudad de México, 2005; 4. Informe de Gobierno: anexo estadístico, Ciudad de México, 2010, y 6. Informe de Gobierno: anexo estadístico, Ciudad de México, 2018.

Este resultado puede interpretarse como sigue. Si la proporción de participación de la población en la fuerza de trabajo se mantiene igual a la tendencia observada de 1988 a 2017, la tasa de crecimiento del ingreso por habitante promedio para el período 2017- 2026 sería del 0,62\%, frente al 1,67\% que se lograría con las políticas propuestas implementadas a partir de 2017. Visto de otro modo, si continúa la tendencia de una mayor participación de la población en el empleo, con las políticas simuladas las tasas de crecimiento del ingreso por habitante fácilmente superarían la cifra del $2 \%$ anual, frente a la registrada de 1988 a 2017, que fue del 1,27\%.

\section{Algunas conclusiones y comentarios finales ${ }^{15}$}

El estancamiento económico de México durante más de 30 años nos lleva a considerar la posibilidad de salir de un "consenso" que ni siquiera en Washington existe ${ }^{16}$. Esta consideración, a su vez, nos conduce a revisar con atención las estrategias económicas seguidas por otros países que han logrado acelerar el crecimiento de sus economías e incrementar el bienestar de sus habitantes en unas cuantas décadas. Estas estrategias, basadas en economías con un sector privado fuerte, pero dirigidas por el Estado, han venido a conocerse como estrategias de Estado desarrollador.

\footnotetext{
${ }^{15}$ En este trabajo se ha planteado como objetivo analizar los resultados que arrojarían dos políticas o dos cambios sencillos que el Gobierno puede impulsar. La matriz de contabilidad social que utilizamos no desagrega la totalidad de los hogares de la economía, lo que permitiría hacer un análisis diferenciado de los distintos patrones de gasto (y de ahorro) que tendrían esos diversos tipos de hogares, según sus patrones de consumo. Esto puede constituir una importante adición futura al modelo, que permitiría llevar a cabo un análisis más completo de las implicaciones para el ahorro y el consumo real.

${ }^{16}$ El Consenso de Washington se refiere a un conjunto de diez recomendaciones de política económica formuladas en 1989 por John Williamson, ofrecidas a los países en desarrollo para salir de la crisis de la deuda de principios de los años ochenta. Entre estas recomendaciones destacan: disciplina fiscal, liberalización del mercado de capitales y divisas, liberalización del comercio internacional, eliminación de obstáculos a la entrada de inversión extranjera directa, privatización de empresas públicas y protección a los derechos de propiedad, incluida la propiedad intelectual (Williamson, 1990).
} 
Como señala Rodrik (2009), la experiencia asiática destaca un aspecto fundamental: una sólida estrategia de desarrollo integral que produce elevado crecimiento económico es mucho más efectiva en lograr la integración con la economía mundial que una estrategia puramente integracionista que se basa en esperar que la mera apertura haga su magia.

Para imaginar las posibilidades de instaurar en México un Estado desarrollador, como se describe en la introducción del presente artículo, se elaboró un MEGA en el que se utilizan solo dos instrumentos del poderoso arsenal que comprende esta estrategia. Simulamos los efectos que tendría en la economía mexicana un subsidio del $20 \%$ a los insumos nacionales otorgado a 12 de 47 sectores considerados claves, y un aumento del ahorro del $10 \%$ al $20 \%$. Con solo estas dos medidas se logra casi duplicar la tasa de crecimiento del ingreso por habitante y, con ello, evitar, o por lo menos contener, la creciente descomposición social. Si al MEGA descrito en este trabajo se agregaran otras medidas, las tasas de crecimiento serían considerablemente mayores.

El propósito de este ejercicio es ilustrar lo que podría hacer el país para salir del estancamiento mediante una nueva estrategia. Este cambio de estrategia obviamente no es sencillo. Existen muchos intereses creados internos y externos, que favorecen el statu quo y son opuestos al cambio y la experimentación.

\section{Bibliografía}

Alfaro, L. y A. Charlton (2013), "Growth and the quality of foreign direct investment", The Industrial Policy Revolution I: The Role of Government Beyond Ideology, J. Stiglitz y J. Lin (eds.), Londres, Palgrave MacMillan.

Amsden, A. (1992), "Getting relative prices 'wrong': a summary", Asia's Next Giant: South Korea and Late Industrialization, Nueva York, Oxford University Press.

Antweiler, W. y D. Trefler (2000), "Increasing returns and all that: a view from trade", NBER Working Paper, № 7941, Cambridge, Oficina Nacional de Investigaciones Económicas (NBER), octubre.

Aoki, M., H. Kim y M. Okuno-Fujiwara (comps.) (2000), El papel del gobierno en el desarrollo económico del Asia Oriental: análisis institucional comparado, Lecturas de El Trimestre Económico, Ciudad de México, Fondo de Cultura Económica (FCE).

Berasaluce, J. y J. Romero (2018), "La política económica exterior coreana (1948-presente): apertura bajo una estrategia nacional", Cambio de la época de Corea y la revisión de su relación con América Latina, W. Suk-Kyun y J. Ramírez (eds.), Ciudad de México, El Colegio de México, en prensa.

Böhm-Bawerk, E. (1998), Teoría positiva del capital, Madrid, Ediciones Aosta.

Castañeda, A. y G. Garduño (2000), "Rendimientos crecientes a escala en la manufactura mexicana", El Trimestre Económico, vol. 67, № 266.

CCO Noticias (2015), "Tener un México más moderno, próspero, justo e incluyente es una responsabilidad compartida: EPN (video)", 1 de abril [en línea] https://corporacioncomunicativaojeda.wordpress.com/2015/04/01/ tener-un-mexico-mas-moderno-prospero-justo-e-incluyente-es-una-responsabilidad-compartida-epn-video/.

Chandrasekhar, C. (2013), "Financialization as an obstacle to industrialization", The Industrial Policy Revolution II: Africa in the 21st Century, J. Stiglitz, J. Lin y E. Patel (eds.), Londres, Palgrave MacMillan.

Chang, H. (2003), Globalisation, Economic Development and the Role of the State, Londres, Third World Network, Zed Books.

Chen, M. (1995), Asian Management Systems: Chinese, Japanese and Korean Styles of Business, Londres, Routledge.

Chibber, V. (2014), "The developmental State in retrospect and prospect: lessons from India and South Korea", The End of the Developmental State?, M. Williams (ed.), Nueva York, Routledge.

Coatsworth, J. (1990), Los orígenes del atraso: nueve ensayos de historia económica de México en los siglos XVIII y XIX, Ciudad de México, Alianza Editorial.

Dixon, P. y B. Parmenter (1996), "Computable general equilibrium modelling for policy analysis and forecasting", Handbook of Computational Economics, vol. 1, H. Amman, D. Kendrick y J. Rust (eds.), Ámsterdam, North-Holland.

Evans, P. (1995), Embedded Autonomy: States and Industrial Transformation, Princeton, Princeton University Press. 
Falck, O., C. Gollier y L. Woessmann (eds.) (2011), Industrial Policy for National Champions, CESifo Seminar Series, Cambridge, MIT Press.

Felipe, J. (ed.) (2015), Development and Modern Industrial Policy in Practice: Issues and Country Experiences, Cheltenham, Banco Asiático de Desarrollo (BAsD), Edward Elgar Publishing.

Greenfeld, L. (2005), Nacionalismo: cinco vías hacia la modernidad, Madrid, Centro de Estudios Políticos y Constitucionales.

Helpman, E. y P. Krugman (1985), Market Structure and Foreign Trade: Increasing Returns, Imperfect Competition, and the International Economy, Cambridge, MIT Press.

Hennings, K. (1990), "Roundabout methods of production", Capital Theory, The New Palgrave, J. Eatwell, M. Milgate y P. Newman (eds.), Londres, Palgrave Macmillan UK.

Hirschman, A. (1968), "La economía política de la industrialización a través de la sustitución de importaciones en América Latina", El Trimestre Económico, vol. 35, № 140.

(1961), La estrategia del desarrollo económico, Ciudad de México, Fondo de Cultura Económica (FCE).

Huff, W. (1995), "The developmental State, government, and Singapore's economic development since 1960", World Development, vol. 23, № 8, agosto.

INEGI (Instituto Nacional de Estadística y Geografía) (2016), "Matriz de Insumo Producto 2012 - actualización. Base 2008" [en línea] https://www.inegi.org.mx/programas/mip12/2008/default.html\#Documentacion.

Johnson, C. (2015), "Instituciones políticas y desempeño económico: la relación entre las empresas y el gobierno en Japón, Corea del Sur y Taiwán", Economía política del crecimiento: cadenas causales y mecanismos institucionales, Serie Estado, Gestión Pública y Desarrollo en América Latina, Buenos Aires, Banco de Desarrollo de América Latina (CAF).

(1982), MITI and the Japanese Miracle: the Growth of Industrial Policy, 1925-1975, Stanford, Stanford University Press.

Kasahara, S. (2013), "The Asian developmental State and the flying geese paradigm", Discussion Papers, № 213 (UNCTAD/OSG/DP/2013/3), Ginebra, Conferencia de las Naciones Unidas sobre Comercio y Desarrollo (UNCTAD), noviembre.

Kim, L. y R. Nelson (eds.) (2000), Technology, Learning, \& Innovation: Experiences of Newly Industrializing Economies, Cambridge, Cambridge University Press.

Kim, Y. (1999), "Neoliberalism and the decline of the developmental State", Journal of Contemporary Asia, vol. $29, N^{\circ} 4$.

Kristof, N. y S. WuDunn (2000), Thunder from the East: Portrait of a Rising Asia, Nueva York, Alfred A. Knopf.

Lee, K. (2013), Schumpeterian Analysis of Economic Catch-up: Knowledge, Path-Creation, and the Middle-Income Trap, Cambridge, Cambridge University Press.

Lee, W. (ed.) (1991), German Industry and German Industrialisation: Essays in German Economic and Business History in the Nineteenth and Twentieth Centuries, Nueva York, Routledge.

Leftwich, A. (1995), "Bringing politics back in: towards a model of the developmental State", The Journal of Development Studies, vol. 31, № 3, febrero.

(1994), "The developmental State", Working Paper, № 6, York, Universidad de York.

Liang, M. (2010), "Confucianism and the East Asian Miracle", American Economic Journal: Macroeconomics, vol. 2, $\mathrm{N}^{\circ} 3$, julio.

Lofgren, H., R. Harris y S. Robinson (2002), A standard computable general equilibrium (CGE) model in Gams, Microcomputers in Policy Research, Washington, D.C., Instituto Internacional de Investigación sobre Políticas Alimentarias (IFPRI).

López-de-Silanes, F., J. Markusen y T. Rutherford (1992), "Complementarity and increasing returns in intermediate inputs: a theoretical and applied general-equilibrium analysis", NBER Working Paper, № 4179, Cambridge, Oficina Nacional de Investigaciones Económicas (NBER).

Malerba, F. y otros (2016), Innovation and the Evolution of Industries: History-Friendly Models, Cambridge, Cambridge University Press.

Maman, D. y Z. Rosenhek (2011), "The institutional dynamics of a developmental State: change and continuity in State-economy relations in Israel", Working Paper Series, № 5, Raanana, Universidad Abierta de Israel.

México (2017), 5. Informe de Gobierno 2016-2017: anexo estadístico, Ciudad de México.

Minns, J. (2001), "Of miracles and models: the rise and decline of the developmental State in South Korea", Third World Quarterly, vol. 22, N 6, diciembre.

Monetary Authority of Singapore (1991), Savings-Investment Balances in Singapore: Determinants and Medium-Term Outlook, Singapur. 
Myrdal, G. (1959), Teoría económica y regiones subdesarrolladas, Ciudad de México, Fondo de Cultura Económica (FCE).

Nabi, I. y J. Shivakumar (2001), Back from the Brink: Thailand's Response to the 1997 Economic Crisis, Washington, D.C., Banco Mundial.

Nelson, R. (2005), Technology, Institutions and Economic Growth, Cambridge, Harvard University Press.

- (1996), The Sources of Economic Growth, Cambridge, Harvard University Press.

Nurkse, R. (1963), "Algunos aspectos internacionales del desarrollo económico", La economía del subdesarrollo, A. Agarwala y S. Singh (eds.), Madrid, Editorial Tecnos.

Önis, Z. (1991), "The logic of the developmental State", Comparative Politics, vol. 24, No 1, octubre.

Partida, V. (2008), Proyecciones de la población económicamente activa de México y de las entidades federativas, 2005-2050, Ciudad de México, Consejo Nacional de Población (CONAPO).

Park, C. (1979), Korea Reborn: A Model for Development, Englewood Cliffs, Prentice-Hall.

Paul, S. (1979), "Transnational corporations and developing countries: some issues in industrial policy", Economic and Political Weekly, vol. 14, No 30/32, agosto.

Permani, R. (2009), "The role of education in economic growth in East Asia: a survey", Asian-Pacific Economic Literature, vol. 23, № 1 , mayo.

Pierenkemper, T. y R. Tilly (2004), The German Economy during the Nineteenth Century, Nueva York, Berghahn Books.

Polidano, C. (2001), "Don't discard State autonomy: revisiting the East Asian experience of development", Political Studies, vol. 49, № 3, agosto.

Prebisch, R. (1960), "La política comercial en los países insuficientemente desarrollados (desde el punto de vista latinoamericano)", Economía, vol. 19, № 69, Santiago, Universidad de Chile.

Rasgotra, M. (ed.) (2013), Science and Technology in China: Implications and Lessons for India, Nueva Delhi, SAGE Publications India.

Rashid, H. (2013), "Does financial market liberalization promote financial development?: evidence from Sub-Saharan Africa", The Industrial Policy Revolution Il: Africa in the 21st Century, J. Stiglitz, J. Lin y E. Patel (eds.), Londres, Palgrave Macmillan.

Rasmussen, P. (1963), Relaciones intersectoriales, Madrid, Aguilar.

Rodríguez, F. y D. Rodrik (1999), "Trade policy and economic growth: a skeptic's guide to the cross-national evidence”, NBER Working Paper, № 7081, Cambridge, Oficina Nacional de Investigaciones Económicas (NBER), abril.

Rodrik, D. (2009), "Trading in illusions", Foreign Policy, 18 de noviembre [en línea] https://foreignpolicy. com/2009/11/18/trading-in-illusions/.

(2007), "The real exchange rate and economic growth: theory and evidence", Working Paper, Boston, Weatherhead Center for International Affairs.

Rosenstein-Rodan, P. (1959), "Problemas de la industrialización de la Europa Oriental y Sudoriental", Revista de Economía Política, № 23.

Sánchez, I. (2011), "Estancamiento económico en México, manufacturas y rendimientos crecientes: un enfoque kaldoriano", Investigación Económica, vol. 70, № 277.

Shin, J. y H. Chang (2003), Restructuring Korea Inc., Londres, RoutledgeCurzon.

Skocpol, T. (2014), "El Estado regresa al primer plano: estrategias de análisis en la investigación actual", Revista Santander, № 9.

Taylor, M. (2016), The Politics of Innovation: Why some Countries are Better than Others at Science and Technology, Nueva York, Oxford University Press.

The Conference Board (2019), "Total Economy Database ${ }^{\mathrm{TM}}$ - Key Findings" [base de datos en línea] http:// www.conference-board.org/data/economydatabase/.

The Nobel Prize (2020), "The increasing returns revolution in trade and geography" [en línea] https://www. nobelprize.org/prizes/economic-sciences/2008/krugman/lecture/.

Thompson, M. (1996), "Late industrialisers, late democratisers: developmental States in the Asia-Pacific", Third World Quarterly, vol. 17, № 4.

Vogel, E. (1991), The Four Little Dragons: The Spread of Industrialization in East Asia, Cambridge, Harvard University Press.

Wade, R. (2003), "What strategies are viable for developing countries today? The World Trade Organization and the shrinking of 'development space'”, Review of International Political Economy, vol. 10, № 4. 
Wan, M. (2008), The Political Economy of East Asia: Striving for Wealth and Power, Washington, D.C., CQ Press. Weiss, L. (2000), "Developmental States in transition: adapting, dismantling, innovating, not 'normalising'”, The Pacific Review, vol. 13, № 1.

Williamson, J. (ed.) (1990), "What Washington means by policy reform", Latin American Adjustment: How Much Has Happened?, Washington, D.C., Peterson Institute for International Economics.

Wong, J. (2004), "The adaptive developmental State in East Asia", Journal of East Asian Studies, vol. 4, $\mathrm{N}^{\circ} 3$, diciembre.

Woo-Cumings, M. (1999), "Introduction: Chalmers Johnson and the politics of nationalism and development", The Developmental State, M. Woo-Cumings (ed.), Ithaca, Cornell University Press.

W Radio (2017), "Quinto Informe de Gobierno de EPN", 2 de septiembre [en línea] http://wradio.com.mx/ radio/2017/09/01/nacional/1504292661_444049.html.

Young, A. (2009), "Rendimientos crecientes y progreso económico, Revista de Economía Institucional, vol. 2, $N^{\circ} 21$.

Zysman, J. (1983), Governments, Markets, and Growth: Financial Systems and the Politics of Industrial Change, Ithaca, Cornell University Press. 


\section{Anexo A1}

\section{Actividades claves de la economía mexicana}

De acuerdo con los índices de Rasmussen, las actividades claves de la economía mexicana, para la matriz de contabilidad social de 2012, con 47 sectores, elaborada en esta investigación, son las que se presentan en el cuadro A1.1.

Cuadro A1.1

Sectores claves según índices de arrastre y dispersión

\begin{tabular}{llcc}
\hline & Descripción & Índice de arrastre & Índice de dispersión \\
\hline A6 & Generación, transmisión y distribución de energía eléctrica & 1,155 & 1,422 \\
\hline A10 & Industria alimentaria & 1,124 & 1,174 \\
\hline A16 & Industria del papel & 1,317 & 1,573 \\
\hline A18 & Fabricación de productos derivados del petróleo y del carbón & 1,316 & 4,196 \\
\hline A19 & Industria química & 1,125 & 4,349 \\
\hline A20 & Industria del plástico y del hule & 1,310 & 1,389 \\
\hline A22 & Industrias metálicas básicas & 1,210 & 2,349 \\
\hline A23 & Fabricación de productos metálicos & 1,360 & 1,448 \\
\hline A24 & Fabricación de maquinaria y equipo & 1,387 & 1,636 \\
\hline A25 & Fabricación de equipo de computación, comunicación, medición y de otros equipos, & 2,032 & 2,892 \\
& componentes y accesorios electrónicos & & 1,324 \\
\hline A26 & Fabricación de accesorios, aparatos eléctricos y equipo de generación de energía eléctrica & 1,576 & 1,473 \\
\hline A27 & Fabricación de equipo de transporte & 1,481 & \\
\hline
\end{tabular}

Fuente: Elaboración propia. 


\section{Anexo A2}

\section{Parámetros y variables del modelo dinámico-recursivo de equilibrio general aplicado (MEGA)}

Cuadro A2.1

Parámetros del Mega-Mx12

\begin{tabular}{|c|c|c|c|}
\hline Parámetro & Descripción & Parámetro & Descripción \\
\hline \multicolumn{4}{|l|}{ Factores } \\
\hline Captotecon & Capital total en la economía & & \\
\hline Trabtotoecon & Trabajo total en la economía & & \\
\hline \multicolumn{4}{|l|}{ Hogares } \\
\hline$\tau^{\text {caphog }}$ & Parte de hogares en captotecon & & \\
\hline PMAHOG & Propensión marginal al ahorro & & \\
\hline$\sigma c p$ & Elasticidad constante de sustitución & Producción & Valor agregado Cobb-Douglas \\
\hline$\delta c p$ & Preferencia del consumidor & $\alpha c a p_{j}$ & Participación del capital \\
\hline Gobierno & & $\alpha t r a b_{j}$ & Participación del trabajo \\
\hline$\tau^{\text {isrhog }}$ & Tasa del ISR de los hogares & aescva $_{j}$ & Parámetro de escala \\
\hline$\tau^{\text {isrcap }}$ & Tasa del ISR de las empresas & & Insumo combinado CES \\
\hline$\tau^{\text {lDhogNAL }}$ & Impuesto a bien de consumo nacional & $\sigma i c_{i, j}$ & Elasticidad de sustitución \\
\hline$\tau^{\text {IDhogRdM }}$ & Impuesto a bien de consumo importado & $\delta i c_{i, j}$ & Participación de los factores \\
\hline$\tau_{j}^{I P}$ & Impuesto a producción por actividad & $\Phi i c_{i, j}$ & Parámetro de escala \\
\hline$\tau^{\text {invrdm }}$ & Impuesto sobre importación de capital & & Oferta total Leontief \\
\hline$\alpha_{\text {trgobhog }}$ & Parte de transferencias en gasto público & ruic $_{i, j}$ & Requerimiento unitario de insumos \\
\hline$\alpha_{\text {ahrgob }}$ & Parte del ahorro en gasto público & ruva $_{j}$ & Requerimiento unitario de valor agregado \\
\hline$\alpha_{\text {conspubrdm }}$ & Parte de importaciones en gasto público & & Bien agregado de consumo final privado \\
\hline$\alpha_{\text {Conspubnal }}$ & Parte de consumo en gasto público & rucp $_{i}$ & Requerimientos unitarios para el bien final \\
\hline$\beta_{\text {CONSPUBi }}$ & Parte de cada bien en gasto público & $R d M$ & \\
\hline Inversión & & $\tau^{\text {caprdm }}$ & Parte del RdM en captotecon \\
\hline$\tau^{\text {deprec }}$ & Tasa de depreciación & $\alpha_{\text {TRABRDM }}$ & Parte del trabajo en el gasto del RdM \\
\hline$\tau^{\text {reinv }}$ & Tasa de reinversión & $\alpha_{\text {TRRDM }}$ & Parte de las remesas en el gasto del RdM \\
\hline$\alpha_{\text {INVRdM }}$ & Parte de importación de capital en inversión total & $\alpha_{A H R R D M}$ & Participación del ahorro en el gasto \\
\hline$\alpha_{I N V N A L}$ & Parte de inversión interna en inversión total & $\alpha_{E X P O R T}$ & $\begin{array}{l}\text { Participación de las exportaciones en el gasto } \\
\text { del RdM }\end{array}$ \\
\hline$\beta_{I N V i}$ & Parte de cada bien en inversión interna & $\beta_{\text {EXPORTi }}$ & Parte de cada bien en las exportaciones \\
\hline
\end{tabular}

Fuente: Elaboración propia.

Nota: ISR: impuesto sobre la renta; CES: elasticidad de sustitución constante (constant elasticity of substitution); RdM: resto del mundo. 
Cuadro A2.2

Variables endógenas del Mega-Mx12

\begin{tabular}{|c|c|c|c|}
\hline Variable & Descripción & Variable & Descripción \\
\hline & HOGARES & & GOBIERNO \\
\hline INGDISP & Ingreso disponible de hogares & RECISR & Recaudación por ISR \\
\hline AHRHOG & Ahorro de hogares & RECIMPPROD & $\begin{array}{l}\text { Recaudación por impuesto a productos } \\
\text { y producción }\end{array}$ \\
\hline PMAHOG & Propensión marginal al ahorro & RECIMPINV & Recaudación por importación de capital \\
\hline CONSPRIVNAL & Consumo privado del bien nacional & INGGOB & Ingresos del Gobierno \\
\hline CONSPRIVRAM & Consumo privado del bien importado & TAUISRHOG & Tasa de ISR pagada por hogares \\
\hline \multirow[t]{2}{*}{ IPCES } & Índice CES & TRGOBHOG & Transferencias sociales \\
\hline & AHORRO-INVERSIÓN & $A H R G O B$ & Ahorro público \\
\hline INVRdM & Inversión en capital importado & CONSPUBi & Consumo del Gobierno \\
\hline INVNALi & Inversión en capital nacional & CONSPUBRdM & Importaciones del Gobierno \\
\hline \multirow[t]{2}{*}{ AHRTOT } & Ahorro total de la economía & SPRVTGOB & Superávit público \\
\hline & PRODUCCIÓN & & \\
\hline DEMCAP $_{j}$ & Demandas de capital & & PRECIOS \\
\hline$D_{\text {DMTRAB }}$ & Demandas de trabajo & Pcap & Precio del capital \\
\hline$V A_{j}$ & Valor agregado & Ptrab & Precio del trabajo \\
\hline DEMINSNAL $_{i, j}$ & Demanda de insumos nacionales & $P v a_{j}$ & Precio del valor agregado \\
\hline DEMINSEXT & Demanda de insumos importados & Pinscombi & Precio del insumo combinado \\
\hline DEMINSCOMBI $I_{i, j}$ & Demandas de insumos combinados & Pot $_{i}$ & Precio de la oferta total \\
\hline OFTOT $_{i}$ & Oferta total por actividad & $P c p$ & Precio del bien agregado de consumo privado \\
\hline \multirow[t]{2}{*}{$D_{E M B y S C P_{i}}$} & Bienes para consumo privado & $T C$ & Tipo de cambio \\
\hline & RESTO DEL MUNDO & $\operatorname{Prdm}_{i}$ & Precio de insumos importados \\
\hline INGRDM & Ingresos del RdM & PrdmCF & Precio de importaciones para consumo final \\
\hline TRRDMHOG & Transferencias del RdM & & \\
\hline AHRRDM & Ahorro del RdM & & \\
\hline TRABRDM & Trabajo contratado por el RdM & & \\
\hline EXPORTi & Exportaciones por actividad & & \\
\hline
\end{tabular}

Fuente: Elaboración propia.

Nota: ISR: impuesto sobre la renta; CES: elasticidad de sustitución constante (constant elasticity of substitution); RdM: resto del mundo. 


\section{Anexo A3}

\section{Modelo matemático}

\section{A. Hogares}

INGDISP $=\left[\tau^{\text {caphog* }}\left[\text { captotecon* } * 1-\tau^{\text {israp }}-\tau^{\text {depr }}-\tau^{\text {reinv }}\right)\right]^{*}$ Pcap + (trabtotecon - TRABRdM)*Ptrab $]^{*}\left(1-\tau^{I S R H O G}\right)+T R A B R d M * P r d m C F * T C+$ $T R G O B H O G+T R R d M H O G * T C$

$$
\begin{aligned}
& \text { CONSPRIVNAL }=\frac{(I N G D I S P-A H R H O G) * \delta_{C P}^{\sigma^{C P}} *\left[P c P^{*}\left(1+\sigma^{I D h o g N A L}\right)\right]^{-\sigma^{C P}}}{I P C E S}
\end{aligned}
$$

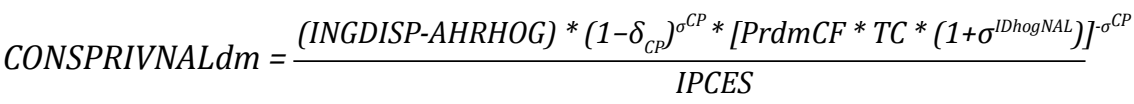

$$
\begin{aligned}
& \text { IPCES }=\delta_{C P}^{\sigma C P} *\left[P C p^{*}\left(1+\sigma^{\text {IDhogNAL }}\right)\right]^{1-\delta C P}+ \\
& \left(1-\delta_{C P}\right)^{\sigma^{C P}} *\left[\operatorname{PrdmCF} * T C *\left(1+\sigma^{\text {IDhogNAL }}\right)\right]^{1-\delta_{C P}} \\
& A H R H O G=P M A H O G * \text { INGDISP }
\end{aligned}
$$

\section{B. Gobierno}

$$
\begin{aligned}
& \text { RECISR }=\left[\tau^{\text {caphog } *} \text { captotecon } *\left(1-\tau^{\text {isrcap }}-\tau^{\text {depr }}-\tau^{\text {reinv }}\right) * \text { Pcap }+\right. \\
& \text { (trabtotecon }- \text { TRABRdM) *Ptrab] }{ }^{*} \tau^{\text {ISRHOG }}+\tau^{\text {israp } *} \text { captotecon * Pcap } \\
& \text { RECIMPPROD }=\sigma^{I D h o g N A L} * \text { CONSPRIVNAL } * P C p+ \\
& \sigma^{I D h o g R d M} * \text { CONSPRIVrdm * PrdmCF * TC + } \\
& \sum_{j}\left[\text { VA }_{j} * \text { Pva }_{j}+\sum_{i} \text { DEMINSCOMBI }_{i, j} * \text { Pinscombi }_{i, j}\right] * \tau_{j}^{I P} \\
& R E C I M P I N V=\tau^{\text {invrdm } *}(I N V R d M * P r d m C F * T C) \\
& I N G G O B=R E C I S R+R E C I M P P R O D+R E C I M P I N V \\
& T R G O B H O G=\alpha^{\text {trgobhog }} * I N G G O B \\
& A H R G O B=\alpha^{\text {ahrgob } *} I N G G O B \\
& \operatorname{CONSPUB}_{i}=\frac{\beta_{i}^{\text {conspub } *} \alpha^{\text {conspubnal } *} I N G G O B}{\operatorname{Pot}_{i}} \\
& \text { CONSPUBRdM }=\frac{\alpha^{\text {conspubrdm } *} I N G G O B}{\operatorname{PrdmCF} * T C} \\
& S P R V T G O B=I N G G O B-T R G O B H O G-A H R G O B- \\
& \text { CONSPUBRdM }{ }^{*} P_{R d m} C F * T C-\sum_{i} C^{*} \text { NSPUB }_{i}{ }^{*} \text { Pot }_{i}
\end{aligned}
$$

\section{Ahorro-inversión}

$$
\begin{gathered}
\text { AHRTOT }=\text { AHRHOG }+ \text { AHRGOB }+ \text { AHRRdM*TC }+ \\
\left(\tau^{\text {depr }}+\tau^{\text {reinv }}\right) * \text { captotecon * Pcap } \\
\text { INVRdM }=\frac{\alpha^{\text {invrdm * }} \text { AHRTOT }}{\operatorname{PrdmCF}^{*} T C^{*}\left(1+\tau^{\text {invrdm }}\right)}
\end{gathered}
$$

Dos propuestas para el cierre ahorro-inversión se incluyen en la sección sobre cierres macroeconómicos. 


\section{Factores productivos}

Véase la sección sobre cierres macroeconómicos.

\section{E. Producción}

Producción Cobb-Douglas del valor agregado. Para la producción del valor agregado (factor compuesto), utilizamos una agregación Cobb-Douglas de rendimientos constantes a escala, de donde la minimización del costo total arroja las demandas óptimas:

$$
\begin{aligned}
\operatorname{DEMCAP}_{J} & =\frac{\alpha c a p_{j}{ }^{*} V A_{j}{ }^{*} P v a_{j}}{P c a p} \\
\operatorname{DEMTRAB}_{J} & =\frac{\alpha \operatorname{trab_{j}}{ }^{*} V A_{j}{ }^{*} P v a_{j}}{P \operatorname{trab}}
\end{aligned}
$$

Con $\alpha c a p_{j}+\alpha t r a b_{j}=1$. Y del supuesto de competencia perfecta:

$$
V A_{j}=\operatorname{aescva}_{j}^{*} D E M C A P_{j}^{\alpha c a p_{j}} * D_{\text {DMTRAB }}^{\alpha t r a b_{j}}
$$

De acuerdo con lo planteado en la sección II, utilizamos para cada sector productivo $j$, la forma funcional:

$$
Y_{t}=\left(\frac{V A_{t}}{V A_{t-1}}\right)^{\mu} \operatorname{aescva}_{t}^{*} D E M C A P_{t}^{\alpha c a p_{j}} * D E M T R A B_{t}^{\alpha t r a b_{j}}
$$

Con $\mu=1,5$ para las actividades claves y $\mu=0$ para las demás.

Producción CES del insumo combinado. Al minimizar el costo las demandas óptimas son:

$$
\begin{aligned}
& \text { DEMINSNAL }_{i, j}=\frac{\text { DEMINSCOMBI }}{\text { fiic }_{i, j}} * \\
& \frac{\delta i c_{i, j}^{\sigma i i_{i j} *} \operatorname{Pot}_{i}^{-\sigma i c_{i, j}}}{\left[\delta i c_{i, j}^{\sigma i c_{i, j} *} \operatorname{Pot}_{i}^{-\sigma i c_{i, j}}+\left(1-\delta i c_{i, j}\right)^{\sigma i c_{i, j} *}\left(\operatorname{Prdm}_{i} * T C\right)^{1-\sigma i c_{i, j}}\right]^{\sigma i c_{i, j} /\left(\sigma i i_{i, j}-1\right)}} \\
& \operatorname{DEMINSEXT~}_{i, j}=\frac{\text { DEMINSCOMBI }_{i, J}}{\text { fiic }_{i, j}} * \\
& \frac{\left(1-\delta i c_{i, j}\right)^{\sigma i c_{i, j} *}\left(\operatorname{Prdm}_{i}{ }^{*} T C\right)^{-\sigma i c_{i, j}}}{\left[\delta i c_{i, j}^{\sigma i c_{i, j} *} \operatorname{Pot}_{i}^{-\sigma i c_{i, j}}+\left(1-\delta i c_{i, j}\right)^{\sigma i c_{i, j} *}\left(\operatorname{Prdm}_{i}{ }^{*} T C\right)^{1-\sigma i c_{i, j}}\right]^{\sigma i i_{i, j} /\left(\sigma i i_{i, j}-1\right)}}
\end{aligned}
$$

Y el supuesto de competencia perfecta:

$$
\begin{aligned}
& \text { Pinscombi }_{i, j}=\frac{1}{\text { fiic }_{i, j}} * \\
& {\left[\delta i c_{i, j}^{\sigma i c_{i, j} *} \operatorname{Pot}_{i}^{1-\sigma i c_{i, j}}+\left(1-\delta i c_{i, j}\right)^{\sigma i i_{i, j} *}\left(\operatorname{Prdm}_{i} * T C\right)^{1-\sigma v a_{i, j}}\right]^{1 /\left(1-\sigma i i_{i, j}\right)}}
\end{aligned}
$$

Producción Leontief de la oferta total (producción total bruta). Al minimizar el costo las demandas óptimas son:

$$
\begin{gathered}
\operatorname{VA}_{j}=\operatorname{ruva}_{j}{ }^{*} \text { OFTOT }_{j} \\
\operatorname{DEMINSCOMBI}_{i, j}=\text { ruic }_{i, j} * \text { OFTOT }_{j}
\end{gathered}
$$


Y por el supuesto de competencia perfecta:

$$
\text { Pot }_{j}=\left[\text { ruva }_{j}{ }^{*} \text { Pva }_{j}+\sum_{i} \text { ruic }_{i, j}{ }^{*} \text { Pinscombi }_{i, j}\right] *\left(1+\tau_{j}^{I P}\right)
$$

Producción Leontief del bien agregado nacional de consumo final privado:

$$
\begin{aligned}
\text { DEMBYSCP }_{i} & =\text { CONSPRIVNAL }^{*} \text { rucp }_{i} \\
\text { Pcp } & =\sum_{i} \text { Pot }_{i}^{*} \text { rucp }_{i}
\end{aligned}
$$

\section{F. $\quad$ Resto del mundo (RdM)}

A precios del RdM.

$$
\begin{aligned}
& I N G R D M=[C O N S P R I V R d M+C O N S P U B R d M+I N V R d M] * P r d m C F \\
& +\sum_{i} \sum_{j} \text { DEMINSEXT }_{i, j} * \operatorname{Prdm}_{i} \\
& +\tau^{\text {caprdm } *} \text { captotecon }\left(1-\tau^{\text {iscap }}-\tau^{\text {deprec }}-\tau^{\text {reinv }}\right) \frac{P_{C A P}}{T C} \\
& \text { TRRDMHOG }=\alpha_{\text {trrdm }} * \text { INGRDM } \\
& A H R R D M=\alpha_{\text {AHRrdm }} * \text { INGRDM } \\
& \text { TRABRDM }=\alpha_{\text {trabrdm }} \frac{I N G R D M}{P_{R D M} C F} \\
& \text { EXPORT }_{i}=\text { Bexport }_{i}^{*} \alpha_{\text {export }} \frac{I N G R D M}{\operatorname{Pot}_{i} / T C}
\end{aligned}
$$

Supuesto de país pequeño:

$$
\begin{gathered}
\operatorname{PrdmCF}=1 \\
\operatorname{Prdm}_{i}=1
\end{gathered}
$$

\section{G. Cierres macroeconómicos}

La suma de las demandas de capital (trabajo) en cada actividad es igual al capital (trabajo) total de la economía (pleno empleo).

$$
\begin{gathered}
\sum_{j} D_{E M C A P_{j}}=\text { captotecon } \\
\sum_{j} D_{E M T R A B_{j}}=\text { trabtotecon }- \text { TRABRdM }
\end{gathered}
$$

Suponiendo que la propensión al ahorro de los hogares no varía significativamente ante cambios relativamente pequeños:

$$
\left[\text { AHRTOT - INVRdM * PrdmCF * TC }\left(1+\tau^{i \text { inrdm }}\right)\right] \beta \text { inv }_{i}=I N V N A L_{i}^{*} \operatorname{Pot}_{i}
$$

Fijando la inversión, queda como variable la propensión marginal al ahorro:

$$
\begin{aligned}
A H R H O G & =\sum_{i} I N V N A L_{i}^{*} \operatorname{Pot}_{i}+I N V R d M * \operatorname{PrdmCF}^{*} T C\left(1+\tau^{\text {invrdm }}\right) \\
& -A H R G O B-\left(\tau^{\text {deprec }}+\tau^{\text {reinv }}\right) * \text { captotecon }{ }^{*} P_{C A P}-A H R R d M * T C
\end{aligned}
$$

Por último, la demanda total de bienes tiene que ser igual a la oferta total:

$$
\text { OFTOT }_{i}=\sum_{j} \text { DEMINSNAL }_{i, j}+\text { DEMBYSCP }_{i}+\text { CONSPUB }_{i}+\text { INV }_{i}+\text { EXPORT }_{i}
$$




\section{H. Dinámica recursiva}

Se realizó el ejercicio para un horizonte de diez períodos $(t=1,2, \ldots, 10)$. Como ya se señaló, el trabajo se actualiza en cada período de acuerdo con la tasa de crecimiento (constante) de la población económicamente activa (PEA).

Con respecto al capital, su rendimiento real $\left(k t r_{t}\right)$ está dado por el valor total de las rentas dividido por el ahorro total, multiplicado por la tasa de crecimiento $g_{t}$. Visto de otro modo: $g_{t}=\left(\right.$ AHRTOT $\left._{t}{ }^{*} k t r_{t}\right)$ $/\left(\right.$ Pcap $_{t} *$ captotecon $\left._{t}\right)$, lo que significa que el ahorro multiplicado por su rendimiento y dividido por las rentas totales arroja la tasa de crecimiento, pues la diferencia entre las rentas de dos períodos constituye el ahorro. De acuerdo con esto, el capital se actualiza en cada período como sigue:

$$
\begin{aligned}
& \text { captotecon }_{t+1}=\left(1+g_{t}\right) * \text { captotecon }_{t} \\
& g_{t}=\text { AHRTOT }_{t} * \text { ktr }_{t} /\left(\text { Pcap }_{t} * \text { captotecon }_{t}\right)
\end{aligned}
$$




\section{Anexo A4}

\section{Actividades y su agregación en grandes sectores}

\begin{tabular}{|c|c|c|}
\hline Gran sector & Número & Actividad \\
\hline \multirow[t]{2}{*}{ Agropecuario } & 1 & Agricultura \\
\hline & 2 & Cría y explotación de animales; aprovechamiento forestal; pesca, caza y captura; servicios relacionados \\
\hline \multirow[t]{3}{*}{ Petróleo y minería } & 3 & Extracción de petróleo y gas \\
\hline & 4 & Minería de minerales metálicos y no metálicos, excepto petróleo y gas \\
\hline & 5 & Servicios relacionados con la minería \\
\hline \multirow[t]{2}{*}{ Electricidad y gas } & 6 & Generación, transmisión y distribución de energía eléctrica \\
\hline & 7 & Suministro de agua y suministro de gas por ductos al consumidor final \\
\hline \multirow[t]{2}{*}{ Construcción } & 8 & Edificación \\
\hline & 9 & Construcción de obras de ingeniería civil; trabajos especializados \\
\hline \multirow[t]{19}{*}{ Manufacturas } & 10 & Industria alimentaria \\
\hline & 11 & Industria de las bebidas y del tabaco \\
\hline & 12 & Insumos y acabados textiles, y productos textiles, excepto prendas de vestir \\
\hline & 13 & Fabricación de prendas de vestir \\
\hline & 14 & Curtido y acabado de cuero y piel, y manufactura de productos de cuero, piel y sucedáneos \\
\hline & 15 & Industria de la madera \\
\hline & 16 & Industria del papel \\
\hline & 17 & Impresión e industrias conexas \\
\hline & 18 & Fabricación de productos derivados del petróleo y del carbón \\
\hline & 19 & Industria química \\
\hline & 20 & Industria del plástico y del hule \\
\hline & 21 & Fabricación de productos a base de minerales no metálicos \\
\hline & 22 & Industrias metálicas básicas \\
\hline & 23 & Fabricación de productos metálicos \\
\hline & 24 & Fabricación de maquinaria y equipo \\
\hline & 25 & Equipo de computación, comunicación, medición y otros electrónicos \\
\hline & 26 & Accesorios, aparatos eléctricos y equipo de generación de energía eléctrica \\
\hline & 27 & Fabricación de equipo de transporte \\
\hline & 28 & Otras industrias manufactureras \\
\hline \multirow[t]{19}{*}{ Servicios } & 29 & Comercio \\
\hline & 30 & Transportes \\
\hline & 31 & Servicios postales, paquetería y almacenamiento \\
\hline & 32 & Información en medios masivos \\
\hline & 33 & $\begin{array}{l}\text { Banca central, instituciones de intermediación crediticia y financiera no bursátil, y actividades bursátiles, } \\
\text { cambiarias y de inversión financiera }\end{array}$ \\
\hline & 34 & Compañías de fianzas, seguros y pensiones \\
\hline & 35 & Servicios inmobiliarios \\
\hline & 36 & $\begin{array}{l}\text { Servicios de alquiler de bienes muebles, y servicios de alquiler de marcas registradas, patentes } \\
\text { y franquicias }\end{array}$ \\
\hline & 37 & Servicios profesionales, científicos y técnicos \\
\hline & 38 & Corporativos \\
\hline & 39 & Apoyo a negocios y manejo de desechos y servicios de remediación \\
\hline & 40 & Servicios educativos \\
\hline & 41 & Servicios médicos de consulta externa y servicios relacionados \\
\hline & 42 & Hospitales; asistencia social e instalaciones de cuidado de la salud; otros servicios de asistencia \\
\hline & 43 & Servicios de esparcimiento culturales y deportivos, y otros servicios recreativos \\
\hline & 44 & Servicios de alojamiento temporal \\
\hline & 45 & Servicios de preparación de alimentos y bebidas \\
\hline & 46 & Otros servicios excepto actividades gubernamentales \\
\hline & 47 & $\begin{array}{l}\text { Actividades legislativas, gubernamentales, de impartición de justicia y de organismos internacionales } \\
\text { y extraterritoriales }\end{array}$ \\
\hline
\end{tabular}

Fuente: Elaboración propia. 